\title{
Fe/O RATIO BEHAVIOR AS AN INDICATOR OF SOLAR PLASMA STATE AT DIFFERENT SOLAR ACTIVITY MANIFESTATIONS AND IN PERIODS OF THEIR ABSENCE
}

\section{G.S. Minasyants}

Fesenkov Astrophysical Institute, Almaty, Kazakhstan, gennadii_minasya@mail.ru

\section{T.M. Minasyants}

Fesenkov Astrophysical Institute,

Almaty, Kazakhstan, gennadii_minasya@mail.ru

\author{
V.M. Tomozov \\ Institute of Solar-Terrestrial Physics SB RAS, \\ Irkutsk,Russia,tom@iszf.irk.ru
}

\begin{abstract}
We report the results of the investigation into plasma physical characteristics at various solar activity manifestations and in periods of their absence. These results have been obtained from quantitative estimates of the relative abundance of $\mathrm{Fe}$ and $\mathrm{O}$ ions in different energy ranges. Maximum values of the $\mathrm{Fe} / \mathrm{O}$ ratio is shown to correspond to particle fluxes from impulsive flares for ions with energies $<2 \mathrm{MeV} / \mathrm{n}$ (the most significant manifestation of the first ionization potential (FIP) effect). In particle fluxes from gradual flares, the $\mathrm{Fe} / \mathrm{O}$ value decreases smoothly with ion energy and is noticeably inferior to values of fluxes in impulsive events. We have established that the properties of flares of solar cosmic rays indicate their belonging to a separate subclass in the total population of gradual events. Relying on Fe/O variations, we propose an explanation of the solar plasma behavior during the development of flares of both classes.

Magnetic clouds (a separate class of coronal mass ejections (CME)), which have turbulent compression regions and are sources of strong geomagnetic storms, exhibit a relative composition of Fe ions comparable to the abundance of Fe in ion fluxes from gradual flares.

We have found out that the $\mathrm{Fe} / \mathrm{O}$ value can be used to detect penetration of energetic flare plasma into the CME body at the initial phase of their joint development and to estimate its relative contribution.
\end{abstract}

During solar minimum with complete absence of sunspots, the $\mathrm{Fe} / \mathrm{O}$ ratio at a distance of 1 AU show absolutely low values of $\mathrm{Fe} / \mathrm{O}=0.004-0.010$ in the energy range from $2-5$ to $30 \mathrm{MeV} / \mathrm{n}$. This is associated with the manifestation of the cosmic ray anomalous component, which causes an increase in the intensity of ion fluxes with a high FIP, including oxygen $(\mathrm{O})$, and elements with a low FIP (Fe) demonstrate weakening of the fluxes. As for particles with higher energies $\left(E_{\mathrm{k}}>30 \mathrm{MeV} / \mathrm{n}\right)$, the $\mathrm{Fe} / \mathrm{O}$ increase is due to the decisive influence of galactic cosmic rays on the composition of admixtures in the solar wind under solar minimum conditions. The abundance of heavy elements in galactic cosmic rays $30-500 \mathrm{MeV} / \mathrm{n}$ is similar to values in fluxes from gradual flares during high solar activity.

During solar minimum without sunspots, the Fe/O behavior for different ion energy ranges in plasma flows from coronal holes $(\mathrm{CH})$ and in the solar wind exhibits only minor deviations. At the same time, plasma flow associated with the disturbed frontal $\mathrm{CH}$ region can be sources of moderate geomagnetic storms.

Keywords solar activity, energy spectra, FIP effect, $\mathrm{Fe} / \mathrm{O}$ ratio.

\section{INTRODUCTION}

The Sun has a wide range of active phenomena different in their characteristics. The most powerful phenomena are solar flares, which often occur with coronal mass ejections (CME). These processes largely determine space weather conditions. By a number of parameters, flares are divided into two classes. Relatively compact and short-term flares are classed as impulsive events; and long-term flares occupying a larger volume of the solar atmosphere, as gradual events. Impulsive flares cause a strong increase in the ratio of isotopes ${ }^{3} \mathrm{He} /{ }^{4} \mathrm{He}$ (relative to coronal values, the increase is $10^{3}$ ), as well as the high ratio $\mathrm{Fe} / \mathrm{O} \sim 1.3$ and high charge states of ions (for example, $Q(\mathrm{Fe})>16$ ), and hence a high temperature in the vicinity of their sources.

The main acceleration of solar energetic particles in the immediate region of flares is direct acceleration by an electric field during magnetic field dissipation in current sheets of an active region in a magnetic reconnection region, which is followed by stochastic acceleration due to the development of various plasma instabilities [Altyntsev et al., 1982; Priest, Forbes, 2005; Somov, 2013].

Gradual flares occur throughout the solar corona due to dynamic reconstruction of the magnetic field structure and exhibit increased emission in the highest energy ranges of the spectrum for several hours. An additional source of particle acceleration associated with the development of high-speed CME may be the occurrence of shock waves in upper coronal layers and in the interplanetary medium. Flareaccelerated particle fluxes in gradual events are depleted in electrons and enriched in energetic protons. In such phenomena, Fe/O 0.1 is lower than that in impulsive events, and charge states of ions $Q(\mathrm{Fe}) \sim 14$ 
corresponding to coronal temperatures $(2 \div 3) 10^{6} \mathrm{~K}$ are smaller [Li, Zank, 2005].

The above characteristic values of some parameters allow us to determine to which class the flares belong [Reames, 1995], although it is currently believed that the division of flares and related particle fluxes into the two classes is somewhat simplistic [Klecker, 2013] because there are a number of common features in some phenomena of different classes.

For solar flares of the above mentioned classes, we have studied the composition of accelerated plasma particles by analyzing energy spectra of $\mathrm{Fe}$ and $\mathrm{O}$ ions in a wide energy range and resulting $\mathrm{Fe} / \mathrm{O}$ values.

The $\mathrm{Fe} / \mathrm{O}$ ratio is a good indicator of the physical state of the medium under study and a measure of the FIP effect the essence of which is as follows. The composition of elements in the photosphere, fairly reliably determined by spectroscopic methods, is completely homogeneous over the entire visible solar surface; however, the abundance of admixtures in structural formations of the solar corona and SW appears to differently depend on FIP relative to their concentrations in the photosphere. It has been established that the admixtures are fractionated according to FIP in the upper solar chromosphere. Elements with low FIP $(<10 \mathrm{eV}-\mathrm{Fe}$, $\mathrm{Mg}, \mathrm{Si}, \mathrm{K}$, etc.) are easily ionized and carried away under the ponderomotive force of Alfvén waves to the upper solar atmosphere [Laming, 2004], where these ions are able to accumulate largely in the central parts of closed magnetic structures of active regions. Alfvén waves are generated at footpoints of these structures under random plasma motions in photospheric layers. Elements with high FIP ( $>10 \mathrm{eV}-\mathrm{C}, \mathrm{N}$, O, etc.) remain neutral and their abundance does not change. The abundance of Fe ions as an element with low FIP $(<10 \mathrm{eV})$ in the upper solar atmosphere is several fold higher, while the $\mathrm{O}$ abundance remains close to the photospheric one because its FIP exceeds $10 \mathrm{eV}$ [Tomozov, 2012; 2013]. In [Reames et al., 1994; Reames, Ng, 2004; Tylka et al., 2005; Wang et al., 2006; Kahler et al., 2012], results have been reported on determination of $\mathrm{Fe} / \mathrm{O}$ values in accelerated particle fluxes from various solar flares. The Fe/O ratio was measured only in one or two ion energy ranges, which is insufficient for the determination of the energy dependence of the ratio. Consequently, it seems important to obtain quantitative estimates of $\mathrm{Fe} / \mathrm{O}$ in a number of energy ranges for various events. This will allow us to find new properties of particle fluxes.

To construct the ion energy spectra, we have used solar observations from spacecraft (SC) ACE/(ULEIS, EPAM, SIS, CRIS), WIND/EPACT/LEMT. The total particle energy range was $0.04-287.23 \mathrm{MeV} / \mathrm{n}$. We have selected seven separate energy ranges with mean values of $0.06,0.23,1.81,5.30,13.00,30.90$, and $75.69 \mathrm{MeV} / \mathrm{n}$. Energy spectra of the $\mathrm{O}$ and $\mathrm{Fe}$ ions in particle fluxes with a time resolution of $1 \mathrm{hr}$ were obtained using options Multi-source spectral plots of energetic particle fluxes on the OMNI Web Plus Browser website [https://omniweb.gsfc.nasa.gov/ftpbrowser/flux_spectr_ $\mathrm{m} . \mathrm{html}]$. Ion energy is expressed in $\mathrm{MeV} / \mathrm{n}$; fluxes, in particles $/\left(\mathrm{s} \cdot \mathrm{cm}^{2} \cdot \mathrm{sr} \cdot \mathrm{MeV} / \mathrm{n}\right)$. Using values of $\mathrm{Fe}$ and $\mathrm{O}$ fluxes, we calculated their abundance in particle fluxes in the seven energy ranges.

The purpose of this paper is to study characteristics of active processes on the Sun, including flares and CME, as well as features of solar plasma composition in the absence of active regions from the $\mathrm{Fe} / \mathrm{O}$ dynamics using near-Earth SC observations.

\section{VARIATIONS IN Fe/O WITH ION ENERGY IN FLARE-INDUCED FLUXES OF ACCELERATED PARTICLES}

\subsection{Disturbed phase of development of flare-induced particle fluxes}

By constructing ion energy spectra, we have studied flare-induced fluxes of energetic particles for 19972007. When scanning the ion energy spectra with a high time resolution of $1 \mathrm{~h}$, Minasyants, Minasyants [2014] managed to reveal a new feature in the development of powerful flare-induced fluxes, namely, the presence of disturbed and undisturbed phases. By the example of the November 2-4, 2003 event, we illustrate the behavior of $\mathrm{H}$, He, $\mathrm{O}$, and $\mathrm{Fe}$ ion spectra during the flare evolution (Figure 1). At 17:00-17:59 UT on November 2, the flare onset corresponds to a noticeable increase in the number of protons at $E_{\mathrm{k}}>\mathrm{MeV} / \mathrm{n}$. After $1 \mathrm{hr}$ (Figure 1, a), the ion energy spectra take on a dome shape with a flux maximum at $E_{\mathrm{k}} \sim(30 \div 40) \mathrm{MeV} / \mathrm{n}$. The intensity increase on November 2 at 21:00-21:59 UT assumes an arc shape with a maximum at $E_{\mathrm{k}} \sim(2 \div 3) \mathrm{MeV} / \mathrm{n}$ (Figure $\left.1, b\right)$. The arc maximum gradually shifts toward lower energies, and on November 4 at 03:00-03:59 UT the lowenergy particle flux increases with a maximum at $E_{\mathrm{k}} \sim(0.3 \div 0.6) \mathrm{MeV} / \mathrm{n}$ (Figure $\left.1, c\right)$. Finally, at 06:0006:59 UT on November 4, the particle flux with $E_{\mathrm{k}}<0.3 \mathrm{MeV} / \mathrm{n}$ increases (Figure 1, $d$ ) - the disturbed phase ends; energy spectra curves become smooth. During the final, quiet phase of decay of the flare-induced flux (up to 20:00-20:59 UT on November 4), the intensity of particles of different energies gradually decreases to background values.

Thus, during the disturbed period, there are shape distortions in the energy spectra (in particular, for $\mathrm{Fe}$ and $\mathrm{O}$ ions) in the form of arc bends. This indicates the arrival of flare accelerated particles. At the beginning of the disturbed period, the highest-energy particles (in this case, hundreds of $\mathrm{MeV} / \mathrm{n}$ ) are recorded first. During the evolution of the spectrum, the characteristic distortions gradually shift toward lower energies (tens and units of $\mathrm{MeV} / \mathrm{n}$ ), thus forming a bending shape of the spectrum up to energies of several tens of $\mathrm{keV}$. The resulting $\mathrm{Fe} / \mathrm{O}$ ratios contain more information about parameters of the acceleration region and interplanetary medium in which accelerated particles propagate. After the disturbed phase, the ion energy spectra during the quiet evolutionary phase of the flare-induced fluxes become quite smooth and their intensities gradually decrease to background values. When constructing an energy spectrum by integrating over the entire lifetime of a flare-induced flux, we can lose information about proper- 


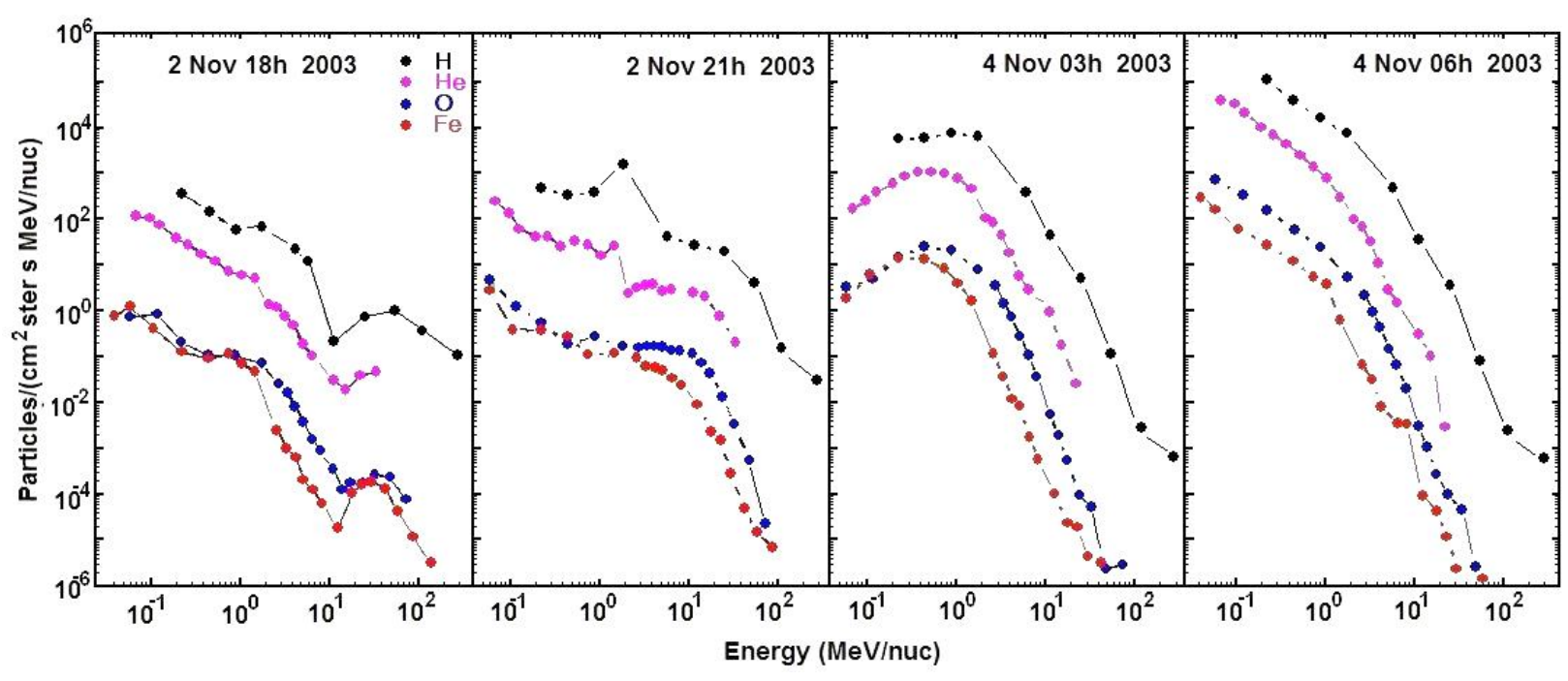

Figure 1. Energy spectra of H, He, O, and Fe ions in flare-induced fluxes on November 2-4, 2003: $a$ - November 2, 18:0018:59 UT; $b$ - November 2, 21:00-21:59 UT; $c$ - November 4, 03:00-03:59 UT; $d$ - November 4, 06:00-6:59 UT

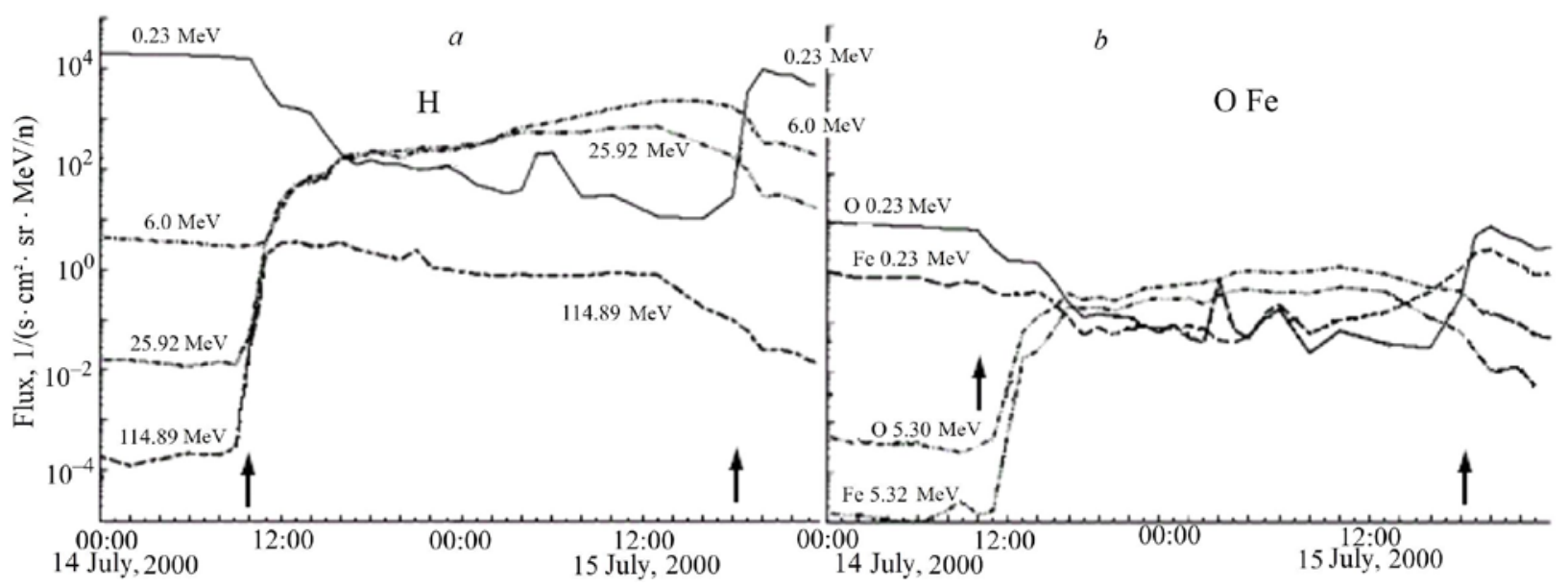

Figure 2. Time profiles of proton (a), O and Fe (b) fluxes during the disturbed phase of the July 14, 2000 event

ties and physical features of acceleration and propagation of flare-induced particles.

Using the July 14, 2000 event as an example (Figure 2), we also examine properties of time profiles of proton $(a), \mathrm{O}$ and $\mathrm{Fe}(b)$ ion fluxes during the disturbed phase of the flare-induced flux. The distribution of time profiles of particle fluxes for different energies is far from typical, especially in a low-energy interval. At the beginning of the disturbed phase, with rapidly increasing highenergy ion flux, the low-energy particle flux decreases. In this case, proton flux values with $E_{\mathrm{k}}=25.92 \mathrm{MeV} / \mathrm{n}$ during the almost entire disturbed phase exceed respective proton intensities with $E_{\mathrm{k}}=0.23 \mathrm{MeV} / \mathrm{n}$.

Distribution of time profiles of $\mathrm{O}$ and $\mathrm{Fe}$ fluxes agrees qualitatively with the proton intensity behavior. A comparison between the $\mathrm{O}$ and Fe profiles (Figure 1, $b$ ) shows that the $\mathrm{Fe} / \mathrm{O}>1$ values are observed in a lowenergy region during most of the disturbed phase. After the disturbed phase ends and the undisturbed phase begins, intensities of low-energy particles abruptly assume typical values. Along the time axis, arrows indicate boundaries of the flux disturbed phase. Such variations in time profiles occur in particle fluxes and other longterm powerful solar energetic particle (SEP) events considered.

\subsection{Fe/O behavior in particle fluxes from strong gradual flares}

The website [http://www.srl.caltech.edu/sampex/DataCenter/DATA/EventSpectra] presents the top 30 SEP events of solar cycle 23. For 16 powerful gradual events included in this list (Table 1) we have obtained Fe/O values calculated from $\mathrm{O}$ and $\mathrm{Fe}$ ion energy spectra in the seven energy ranges during the disturbed phase of flare-induced particle fluxes (Figure 3).

To compare them with typical cases, we have separately examined 16 long-term GLE events - solar cosmic ray bursts during solar cycle 23 (1997-2006).

In these events, increases in relativistic protons are recorded by a network of neutron monitors at ground stations. The $\mathrm{Fe} / \mathrm{O}$ values were also found using the energy spectra of these elements, constructed for the disturbed phase of flare-induced fluxes. Variations in Fe/O for GLE 
Calculated $\mathrm{Fe} / \mathrm{O}$ values for 16 gradual events in 7 ranges of ion energy spectrum

\begin{tabular}{|c|c|c|c|c|c|c|c|c|}
\hline \multirow[t]{2}{*}{ No. } & \multirow[b]{2}{*}{$\begin{array}{l}\text { Periods of the disturbed phase } \\
\text { of particle fluxes in gradual } \\
\text { events }\end{array}$} & \multicolumn{7}{|c|}{$\mathrm{Fe} / \mathrm{O}$} \\
\hline & & $\begin{array}{c}0.06 \\
\mathrm{MeV} / \mathrm{n}\end{array}$ & $\begin{array}{c}0.23 \\
\mathrm{MeV} / \mathrm{n}\end{array}$ & $\begin{array}{c}1.18 \\
\mathrm{MeV} / \mathrm{n}\end{array}$ & $\begin{array}{c}5.30 \\
\mathrm{MeV} / \mathrm{n}\end{array}$ & $\begin{array}{c}13.00 \\
\mathrm{MeV} / \mathrm{n}\end{array}$ & $\begin{array}{c}30.90 \\
\mathrm{MeV} / \mathrm{n}\end{array}$ & $\begin{array}{c}75.69 \\
\mathrm{MeV} / \mathrm{n}\end{array}$ \\
\hline 1 & 12:00.20.04-04:00.23.04.1998 & 0.336 & 0.308 & 0.781 & 0.211 & 0.026 & 0.012 & 0.064 \\
\hline 2 & 12:00.30.09-07:00.02.10.1998 & 0.489 & 0.218 & 0.104 & 0.216 & 0.209 & 0.198 & 0.267 \\
\hline 3 & 06:00.14.11-06:00.15.11.1998 & - & - & - & 0.605 & 0.550 & 0.679 & 0.723 \\
\hline 4 & 23:00.08.11-06:00.10.11.2000 & 0.333 & 0.325 & 0.285 & 0.220 & 0.089 & 0.007 & 0.005 \\
\hline 5 & 06:00.24.11-09:00.26.11.2000 & 0.457 & 0.263 & 0.196 & 0.185 & 0.156 & 0.137 & 0.229 \\
\hline 6 & 22:00.02.04-14:00.04.04.2001 & 0.397 & 0.209 & 0.320 & 0.370 & 0.287 & 0.255 & 0.254 \\
\hline 7 & 08:00.10.04-13:00.11.04.2001 & 0.491 & 0.384 & 0.109 & 0.190 & 0.161 & 0.113 & 0.077 \\
\hline 8 & 01:00.16.08-09:00.17.08.2001 & 0.538 & 0.197 & 0.192 & 0.211 & 0.127 & 0.086 & 0.129 \\
\hline 9 & 11:00.24.09-11:00.27.09.2001 & 0.503 & 0.443 & 0.155 & 0.125 & 0.051 & 0.015 & 0.006 \\
\hline 10 & 13:00.01.10-11:00.03.10.2001 & 0.385 & 0.196 & 0.069 & 0.068 & 0.013 & 0.023 & 0.250 \\
\hline 11 & 21:00.22.11-08:00.24.11.2001 & 0.538 & 0.332 & 0.265 & 0.100 & 0.063 & 0.031 & 0.069 \\
\hline 12 & 00:00.21.04-21:00.22.04.2002 & 0.853 & 0.656 & 0.420 & 0.135 & 0.059 & 0.017 & 0.037 \\
\hline 13 & 21:00.04.11-23:00.07.11.2003 & 0.747 & 1.286 & 0.221 & 0.074 & 0.033 & 0.037 & 0.178 \\
\hline 14 & 18:00.07.11-15:00.09.11.2004 & 0.278 & 0.144 & 0.167 & 0.082 & 0.045 & 0.042 & 0.127 \\
\hline 15 & 20:00.07.09-00:00.13.09.2005 & 0.338 & 0.292 & 0.101 & 0.061 & 0.042 & 0.048 & 0.063 \\
\hline 16 & 18:00.21.04-21:00.22.04.2002 & 0.251 & 0.143 & 0.164 & 0.089 & 0.087 & 0.084 & 0.065 \\
\hline & Mean values & $\begin{array}{l}0.463 \pm \\
0.043\end{array}$ & $\begin{array}{l}0.360 \pm \\
0.074\end{array}$ & $\begin{array}{l}0.237 \pm \\
0.046\end{array}$ & $\begin{array}{l}0.184 \pm \\
0.035\end{array}$ & $\begin{array}{l}0.125 \pm \\
0.034\end{array}$ & $\begin{array}{l}0.111 \pm \\
0.042\end{array}$ & $\begin{array}{l}0.159 \pm \\
0.044\end{array}$ \\
\hline & Median values & 0.457 & 0.292 & 0.192 & 0.160 & 0.075 & 0.045 & 0.102 \\
\hline
\end{tabular}

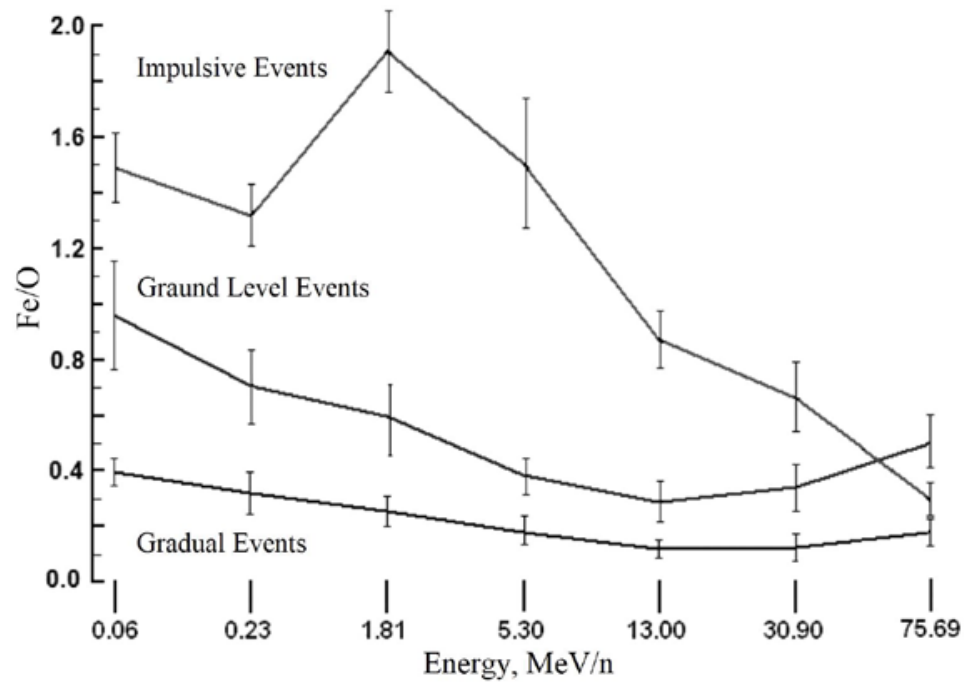

Figure 3. Behavior of the Fe/O ratio in flare-induced particle fluxes for 37 impulsive events, 16 GLE flares, and 16 gradual events. Vertical segments mark measurement errors

fluxes and typical gradual events exhibit both quantitative and qualitative differences (Figure 3). For example, $\mathrm{Fe} / \mathrm{O}$ for GLE decreases with energies increasing to 10.7-15.8 MeV/n, and then noticeably increases, which is not observed in gradual events. This suggests that GLE events may belong to a separate subclass of gradual events.

The most characteristic differences in the behavior of mean Fe/O values for GLE and powerful gradual events were revealed in the energy ranges with mean values of 13.0 and $75.69 \mathrm{MeV} / \mathrm{n}$. We compared the $\mathrm{Fe} / \mathrm{O}$ values for each of the events in energy ranges 10.7-15 and 63.8-89.9 MeV/n and revealed that in the majority of gradual events the $\mathrm{Fe} / \mathrm{O}$ ratio remains practically constant if energy changes. The remaining phe- nomena exhibit both an increase and a decrease in $\mathrm{Fe} / \mathrm{O}$. About $88 \%$ of GLEs display higher Fe/O values; in half of GLE events, $\mathrm{Fe} / \mathrm{O}$ has increased values from 0.58 to 0.97 at $75.69 \mathrm{MeV} / \mathrm{n}$. These distinctive features of the $\mathrm{Fe} / \mathrm{O}$ behavior in the flare-induced GLE fluxes confirm that they can belong to a separate subclass of gradual events [Minasyants et al., 2016].

\subsection{Behavior of the $\mathrm{Fe} / \mathrm{O}$ ratio in particle fluxes from impulsive flares}

Of the whole list of impulsive events [Nitta et al., 2006], we have chosen 37 flares with X-ray importance exceeding $\mathrm{C} 1$ for the subsequent analysis; in particle fluxes from these flares, $\mathrm{Fe} / \mathrm{O}>0.5$ at $2-3 \mathrm{MeV} / \mathrm{n}$. 
Table 2

Calculated $\mathrm{Fe} / \mathrm{O}$ values for 16 bursts of solar cosmic rays in 7 ranges of ion energy spectrum

\begin{tabular}{|c|c|c|c|c|c|c|c|c|}
\hline \multirow[b]{2}{*}{ No. } & \multirow{2}{*}{$\begin{array}{c}\text { Periods of } \\
\text { disturbed phase of GLE particle } \\
\text { fluxes }\end{array}$} & \multicolumn{7}{|c|}{$\mathrm{Fe} / \mathrm{O}$} \\
\hline & & $\begin{array}{c}0.06 \\
\mathrm{MeV} / \mathrm{n}\end{array}$ & $\begin{array}{c}0.23 \\
\mathrm{MeV} / \mathrm{n}\end{array}$ & $\begin{array}{c}1.81 \\
\mathrm{MeV} / \mathrm{n}\end{array}$ & $\begin{array}{c}5.30 \\
\mathrm{MeV} / \mathrm{n}\end{array}$ & $\begin{array}{c}13.00 \\
\mathrm{MeV} / \mathrm{n}\end{array}$ & $\begin{array}{c}30.90 \\
\mathrm{MeV} / \mathrm{n}\end{array}$ & $\begin{array}{c}75.69 \\
\mathrm{MeV} / \mathrm{n}\end{array}$ \\
\hline 1 & 12:00.06.11-02:00.07.11.1997 & - & - & 0.692 & 0.604 & 0.724 & 0.903 & 0.897 \\
\hline 2 & 14:00.02.05-22:00.02.05.1998 & 0.928 & 0.759 & 1.010 & 0.903 & 0.685 & 0.551 & 0.662 \\
\hline 3 & 08:00.06.05-21:00.06.05.1998 & 0.811 & 1.554 & 1.095 & 0.773 & 0.552 & 0.437 & 0.322 \\
\hline 4 & 23:00.24.08-17:00.25.08.1998 & 0.443 & 0.245 & 0.121 & 0.079 & 0.054 & 0.235 & 0.584 \\
\hline 5 & 10:00.14.07-18:00.15.07.2000 & 0.516 & 0.387 & 1.930 & 0.429 & 0.149 & 0.070 & 0.199 \\
\hline 6 & 14:00.15.04-14:00.16.04.2001 & 2.891 & 1.891 & 0.964 & 0.459 & 0.459 & 0.722 & 0.972 \\
\hline 7 & 02:00.18.04-19:00.18.04.2001 & 0.660 & 0.348 & 0.170 & 0.274 & 0.158 & 0.268 & 0.751 \\
\hline 8 & 17:00.04.11-08:00.06.11.2001 & 0.540 & 0.257 & 0.229 & 0.285 & 0.095 & 0.045 & 0.102 \\
\hline 9 & 05:00.26.12-10:00.27.12.2001 & 2.281 & 1.158 & 0.352 & 0.267 & 0.303 & 0.531 & 0.878 \\
\hline 10 & 01:00.24.08-22:00.24.08.2002 & 0.761 & 0.789 & 0.480 & 0.191 & 0.144 & 0.486 & 0.925 \\
\hline 11 & 11:00.28.10-09:00.29.10.2003 & 0.851 & 0.522 & 0.539 & 0.454 & 0.083 & 0.011 & 0.025 \\
\hline 12 & 21:00.29.10-21:00.30.10.2003 & 1.147 & 0.689 & 0.616 & 0.345 & 0.158 & 0.113 & 0.216 \\
\hline 13 & 17:00.02.11-06:00.04.11.2003 & 0.270 & 0.500 & 0.301 & 0.078 & 0.036 & 0.051 & 0.195 \\
\hline 14 & 10:00.17.01-08:00.18.01.2005 & 0.743 & 0.388 & 0.323 & 0.160 & 0.052 & 0.013 & 0.095 \\
\hline 15 & 07:00.20.01-19:00.21.01.2005 & 0.283 & 0.283 & 0.130 & 0.199 & 0.206 & 0.197 & 0.224 \\
\hline \multirow[t]{3}{*}{16} & 03:00.13.12-03:00.14.12.2006 & 1.278 & 0.846 & 0.580 & 0.663 & 0.755 & 0.840 & 0.936 \\
\hline & Mean values & $\begin{array}{l}0.960 \pm \\
0.188\end{array}$ & $\begin{array}{l}0.708 \pm \\
0.126\end{array}$ & $\begin{array}{l}0.596 \pm \\
0.118\end{array}$ & $\begin{array}{l}0.385 \pm \\
0.061\end{array}$ & $\begin{array}{l}0.288 \pm \\
0.065\end{array}$ & $\begin{array}{l}0.342 \pm \\
0.076\end{array}$ & $\begin{array}{l}0.499 \pm \\
0.089\end{array}$ \\
\hline & Median values & 0.761 & 0.522 & 0.510 & 0.315 & 0.158 & 0.252 & 0.458 \\
\hline
\end{tabular}

The origin of the particle fluxes from these impulsive events is probably related to the particle acceleration mechanism in the immediate region of flare energy release. This can be explained by the fact that the CME that accompanied the impulsive flares had neither CMEs with halos nor shock fronts in near-Earth observations. Thus, in view of the possibility of shock-wave acceleration, these ejections were insufficiently effective. Table 3 lists $\mathrm{Fe} / \mathrm{O}$ values for 37 impulsive events in the seven ranges of the energy spectrum. The Fe/O ratio for impulsive and powerful gradual events in the same ion energy ranges is compared in Figure 3. It should be emphasized that a change in the $\mathrm{Fe} / \mathrm{O}$ ratio indicates that the use of a single energy range is not entirely correct in comparing particle fluxes from flare events of different classes. Despite flare-to-flare variations in characteristics of particle fluxes, there is a systematic trend in $\mathrm{Fe} / \mathrm{O}$ values as a function of ion energies. At the same time, processing of the energy spectra when integrating over the entire period of the existence of a flare-induced flux leads to a significant decrease in $\mathrm{Fe} / \mathrm{O}$ values, especially in the region of low ion energies $<2 \mathrm{MeV} / \mathrm{n}$.

\subsection{Discussion and analysis of the $\mathrm{Fe} / \mathrm{O}$ behavior in particle fluxes from flares of differ- ent classes}

Let us now turn to the analysis of the results to qualitatively explain the $\mathrm{Fe} / \mathrm{O}$ behavior in the gradual and impulsive events shown in Figure 3 [Minasyants et al., 2016c].

The classification of flares, elaborated at present, was first proposed by Pallavicini et al. [1977]. According to this classification, impulsive flares occur in small, $<10 \mathrm{Mm}$ high, compact magnetic loops filled with hot plasma with an energy density up to $\sim 10^{3} \mathrm{erg} / \mathrm{cm}^{3}$, and often produce narrow plasma ejections.

Gradual flare events develop in extended diffuse loop-like coronal structures $>50 \mathrm{Mm}$ high with much lower energy density in the flare volume $-<10^{2} \mathrm{erg} / \mathrm{cm}^{3}$. Energy release during flares is observed at loop tops. Gradual flares are closely related to the activation and eruption of filaments - prominences that exist in complex magnetic structures. These flares produce extensive high-energy CMEs. A detailed review of comparative characteristics of impulsive and gradual flares is given in [Kallenrode, 2003] (see also [Cliver et al., 2009]). It is shown that impulsive flares appear in regions of the solar atmosphere with a strong magnetic field and in relatively small volumes. Gradual events occur in complex extended coronal structures with a weaker magnetic field and occupy a much larger volume of the corona.

Wang et al. [2006] have examined characteristics and localization of solar sources of 25 impulsive flares enriched in ${ }^{3} \mathrm{He}$ over a period from 1997 to 2003 (a simplified scheme of magnetic topology of an impulsive flare is shown in Figure 4, $a$ [Kallenrode, 2003]). They have established that sources of the impulsive phenomena are located near the boundaries of $\mathrm{CH}$ with earthward open magnetic field lines. The average distance between the source of accelerated particles and $\mathrm{CH}$, averaged over all the events, was about $4^{\circ}$ in the photosphere. The source was usually a small flare-active region in the W25-W72 longitude range. Note that the presence of numerous small and rapidly evolving active regions located on the boundary of the extensive $\mathrm{CH}$, observed at the Skylab station, was indicated in [Kasinsky, Tomozov, 1974]. In addition, Wang et al. [2006] have found that recurrent plasma ejections along open magnetic field lines were often observed within the estimated interval of injection of fast particles 
Calculated $\mathrm{Fe} / \mathrm{O}$ values for 37 impulsive events in 7 ranges of ion energy spectrum

\begin{tabular}{|c|c|c|c|c|c|c|c|c|}
\hline \multirow[b]{2}{*}{ No } & \multirow[b]{2}{*}{$\begin{array}{l}\text { Periods of development of particle } \\
\text { fluxes in impulsive events }\end{array}$} & \multicolumn{7}{|c|}{$\mathrm{Fe} / \mathrm{O}$} \\
\hline & & $\begin{array}{c}0.06 \\
\mathrm{MeV} / \mathrm{n}\end{array}$ & $\begin{array}{c}0.23 \\
\mathrm{MeV} / \mathrm{n}\end{array}$ & $\begin{array}{c}1.81 \\
\mathrm{MeV} / \mathrm{n}\end{array}$ & $\begin{array}{c}5.30 \\
\mathrm{MeV} / \mathrm{n}\end{array}$ & $\begin{array}{c}13.00 \\
\mathrm{MeV} / \mathrm{n}\end{array}$ & $\begin{array}{c}30.90 \\
\mathrm{MeV} / \mathrm{n}\end{array}$ & $\begin{array}{c}75.69 \\
\mathrm{MeV} / \mathrm{n}\end{array}$ \\
\hline 1 & 03:00.15.08-02:00.17.08.1998 & 0.989 & 1.153 & 1.298 & 0.267 & 0.033 & 0.048 & 0.074 \\
\hline 2 & 11:00.27.09-02:00.28.09.1998 & 1.253 & 1.187 & 1.034 & 1.243 & 0.599 & 0.118 & 0.215 \\
\hline 3 & 01:00.28.09-03:00.29.09.1998 & 1.553 & 1.501 & 1.229 & 1.611 & 0.790 & 0.123 & 0.109 \\
\hline 4 & 07:00.29.09-00:00.30.09.1998 & 1.710 & 1.503 & 1.397 & 1.016 & 0.385 & 0.125 & 0.181 \\
\hline 5 & 06:00.20.02-16:00.20.02.1999 & 0.324 & 0.362 & 2.450 & 6.293 & - & 0.248 & - \\
\hline 6 & 17:00.20.02-10:00.21.02.1999 & 0.465 & 1.076 & 3.807 & 2.481 & 1.069 & 0.328 & 0.081 \\
\hline 7 & $13: 00.21 .02-06: 00.22 .02 .1999$ & 0.684 & 1.227 & 3.575 & - & - & 0.183 & 0.073 \\
\hline 8 & 02:00.11.03-16:00.12.03.1999 г. & 0.784 & 0.689 & 1.318 & 0.530 & 0.276 & 0.516 & 0.107 \\
\hline 9 & 13:00.20.07-10:00.21.07.1999 & 1.054 & 1.566 & 1.344 & 0.216 & 0.541 & 0.141 & 0.092 \\
\hline 10 & 07:00.27.12-05:00.29.12.1999 & 1.384 & 2.216 & 3.953 & 1.342 & 0.619 & 0.112 & 0.207 \\
\hline 11 & $11: 00.07 .03-14: 00.07 .03 .2000$ & 1.967 & 1.801 & 1.042 & 2.007 & 1.824 & 0.741 & 0.178 \\
\hline 12 & $15: 00.07 .03-02: 00.08 .03 .2000$ & 1.895 & 2.882 & 2.006 & 2.158 & 2.052 & 0.981 & 0.163 \\
\hline 13 & 03:00.08.03-18:00.08.03.2000 & 3.012 & 2.559 & 1.033 & 1.625 & 1.245 & 0.595 & 0.062 \\
\hline 14 & $23: 00.18 .03-14: 00.19 .03 .2000$ & 1.153 & 0.418 & 1.986 & 2.043 & - & - & 0.152 \\
\hline 15 & 04:00.23.04-10:00.24.04.2000 & 0.433 & 0.254 & 1.979 & 0.405 & 0.567 & 0.365 & 0.072 \\
\hline 16 & $22: 00.24 .04-16: 00.25 .04 .2000$ & 2.630 & 2.312 & 0.956 & 0.305 & 0.231 & 0.279 & 0.213 \\
\hline 17 & 11:00.01.05-08:00.02.05.2000 & 1.543 & 1.922 & 1.324 & 2.020 & 0.876 & 0.510 & 0.426 \\
\hline 18 & 14:00.04.05-02:00.06.05.2000 & 0.840 & 1.157 & 2.238 & 1.483 & 0.937 & 0.122 & 0.219 \\
\hline 19 & 01:00.24.05-00:00.25.05.2000 & 1.405 & 1.130 & 1.417 & 1.412 & 0.735 & 0.420 & 0.546 \\
\hline 20 & 03:00.22.08-18:00.22.08.2000 & 2.849 & 0.641 & 1.701 & 0.254 & - & - & - \\
\hline 21 & 19:00.22.08-23:00.22.08.2000 & 1.030 & 2.308 & 1.008 & 1.370 & - & - & - \\
\hline 22 & 08:00.27.09-22:00.28.09.2000 & 1.584 & 0.589 & 1.005 & 1.009 & 1.110 & 0.961 & 0.108 \\
\hline 23 & 08:00.29.09-15:00.30.09.2000 & 1.232 & 0.840 & 1.238 & 0.476 & 0.535 & 0.399 & 0.126 \\
\hline 24 & 18:00.14.04-13:00.15.04.2001 & 1.800 & 1.624 & 1.622 & 1.558 & 1.432 & 0.535 & 0.180 \\
\hline 25 & 12:00.08.09-16:00.10.09.2001 & 1.830 & 0.856 & 1.273 & 0.472 & 0.375 & 0.722 & 0.460 \\
\hline 26 & 14:00.11.09-23:00.12.09.2001 & 2.834 & 2.038 & 1.948 & 1.761 & 1.022 & 1.018 & 0.246 \\
\hline 27 & $10: 00.22 .09-23: 00.22 .09 .2001$ & 2.504 & 1.168 & 1.253 & 0.759 & - & - & 0.222 \\
\hline 28 & 16:00.25.02-19:00.26.02.2002 & 1.046 & 1.098 & 1.676 & 0.654 & 0.655 & 0.598 & 0.221 \\
\hline 29 & 14:00.14.04-23:00.14.04.2002 & 1.864 & 1.814 & 3.620 & 6.419 & 0.339 & 0.748 & 0.182 \\
\hline 30 & 00:00.15.04-04:00.15.04.2002 & 1.395 & 1.247 & 2.512 & 0.600 & 0.667 & 1.214 & 0.746 \\
\hline 31 & 05:00.15.04-03:00.16.04.2002 & 1.372 & 1.520 & 2.158 & 1.151 & 0.116 & 0.089 & 0.038 \\
\hline 32 & 23:00.03.08-15:00.04.08.2002 & 2.357 & 1.521 & 2.028 & 1.348 & 0.954 & 0.494 & 0.183 \\
\hline 33 & 16:00.04.08-04:00.06.08.2002 & 2.333 & 0.871 & 2.366 & 1.418 & 1.523 & 3.294 & 0.887 \\
\hline 34 & 11:00.19.08-22:00.19.08.2002 & 0.702 & 0.380 & 1.581 & 1.636 & 1.857 & 1.863 & 0.981 \\
\hline 35 & $23: 00.19 .08-10: 00.20 .08 .2002$ & 1.056 & 0.746 & 2.577 & 2.520 & 1.829 & 1.746 & 1.046 \\
\hline 36 & 11:00.20.08-03:00.22.08.2002 & 1.359 & 1.297 & 3.494 & 2.115 & 1.733 & 1.485 & 1.029 \\
\hline 37 & $15: 00.12 .12-02: 00.13 .12 .2002$ & 0.896 & 1.207 & 2.418 & 0.377 & 0.204 & 0.693 & 0.466 \\
\hline & Mean values & $\begin{array}{l}1.490 \pm \\
0.116\end{array}$ & $\begin{array}{l}1.316 \pm \\
0.105\end{array}$ & $\begin{array}{l}1.910 \pm \\
0.139\end{array}$ & $\begin{array}{l}1.510 \pm \\
0.228\end{array}$ & $\begin{array}{l}0.875 \pm \\
0.102\end{array}$ & $\begin{array}{l}0.661 \pm \\
0.116\end{array}$ & $\begin{array}{l}0.303 \pm \\
0.057\end{array}$ \\
\hline & Median values & 1.384 & 1.207 & 1.676 & 1.359 & 0.735 & 0.510 & 0.183 \\
\hline
\end{tabular}

in extreme ultraviolet (EUV) images of sources. In some relatively rare cases, corresponding mass ejections were traced in white light up to large heliocentric distances. It is important to emphasize that dynamics of these ejections is reflected in time variations of ${ }^{3} \mathrm{He}$ and Fe ion intensities measured with the ACE/ULEIS satellite in an energy range $0.32-0.45 \mathrm{MeV}$. These ejections recorded by $\mathrm{SOHO} / \mathrm{EIT}$ were sometimes followed by $\mathrm{CME}$ observed with the LASCO-C2 coronagraph $\left(\sim(2 \div 6) R_{\odot}\right.$ field of view), which were narrow $\sim 30^{\circ}$ and fast, with velocities of 500 to $1000 \mathrm{~km} / \mathrm{s}$ [Wang et al., 2006].

Wang et al. [2006] have concluded that impulsive flare-induced particle fluxes appear near the boundary between active regions and adjacent $\mathrm{CHs}$ and arise from exchange reconnection between regions of closed and open magnetic fields. During this reconnection, plasma and accelerated particles are transferred from closed to open field lines and are ejected into the heliosphere, followed by narrow collimated plasma ejections. In this scenario, particles accelerate and elements are fractionated in the reconnection region at increased values of the ${ }^{3} \mathrm{He} /{ }^{4} \mathrm{He}$ and $\mathrm{Fe} / \mathrm{O}$ ratios. The magnetic reconnection region can be enriched in ions of heavy elements during a flare due to strong "evaporation" of the chromosphere, when fluxes of accelerated energetic electrons penetrate down into the chromosphere, locally heat it to high tem 


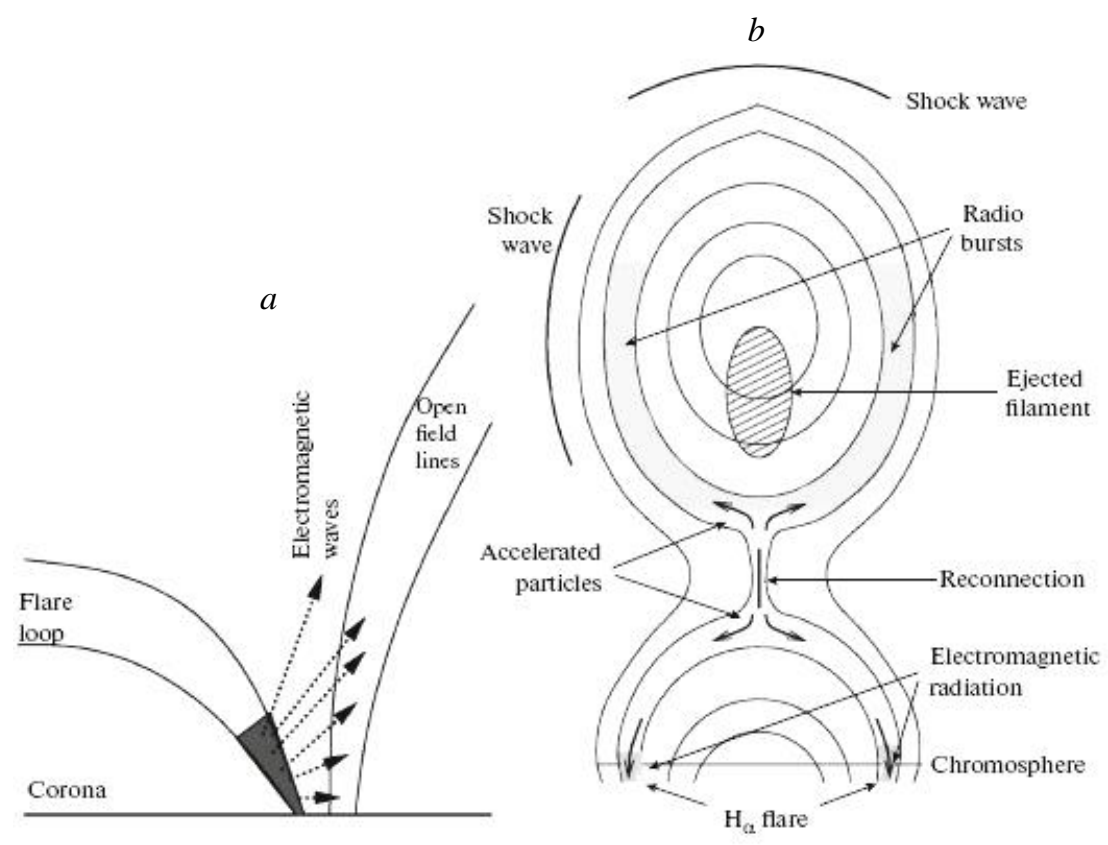

Figure 4. Magnetic structures and particle acceleration zones generating impulsive (a) and gradual $(b)$ flare-induced ion fluxes [Kallenrode, 2003; Cliver et al., 2009]

peratures, and cause a rapid rise of hot plasma. Under these conditions, energetic electrons with a nonthermal distribution function will "extract" some of the cooler ions, thus accelerating them to $1-10 \mathrm{MeV}$ [Mordvinov et al., 1985]. To explain the strong increase in the ${ }^{3} \mathrm{He} /{ }^{4} \mathrm{He}$ ratio (as well as heavy ions from $\mathrm{O}$ to $\mathrm{Fe}$ ) in impulsive flares, a selective acceleration mechanism has been proposed which is associated with the excitation of electromagnetic ion cyclotron waves by energetic electron fluxes [Roth, Temerin, 1998].

Nitta et al. [2006], who search for sources of impulsive events on the Sun and identify their magnetic relationships with Earth, have tried to determine regions of sources of 117 phenomena of flare energetic ions in energy range $2-3 \mathrm{MeV} / \mathrm{n}$. For this purpose, the authors used information about type III radio bursts, which occurred within a 5-hour interval preceding the appearance of the ion fluxes. Thus, they have identified solar sources of 69 of the 117 phenomena. Moreover, in full-disk EUV images, the authors detected ejections in the vicinity of the source shortly after the type III burst. However, it proved difficult to determine the magnetic relationship with Earth, and only in $40 \%$ of the cases the field lines close to the source region in the photosphere were near the coordinates of the Parker spiral on the surface of the source. It should be emphasized that structures with an open magnetic field have also been found in $80 \%$ of the source regions near them [Nitta et al., 2006].

This allows us to draw a preliminary conclusion that the $\mathrm{Fe} / \mathrm{O}$ peak in the energy dependence (Figure 3 ) is caused by $\mathrm{Fe}$ ions accelerated in the region of the impulsive flare. Of crucial importance is the presence of magnetic structures with open field lines going into the interplanetary medium (e.g., $\mathrm{CH}$ ) in the vicinity of flareactive regions (Figure $4, a$ ). The process providing the acceleration of ions and their release into the interplane- tary medium is the exchange reconnection of magnetic fields at the boundary between closed and open magnetic fields in the chromosphere of active regions. Fe ions with low FIP subject to the ponderomotive force of Alfvén waves can be accumulated in the central parts of the active regions [Laming, 2004].

Gradual flare events with particle acceleration by shock waves occur in complex magnetic structures (Figure $4, b$ ) containing a magnetic flux filament above the neutral line in the photosphere. When the general equilibrium of the magnetic field configuration is disturbed, the filament loses stability and starts to rapidly raise upward, thus causing reconnection of the magnetic field in its lower part and initiating the occurrence of a flare process (Figure 4, b). CME (a filament is in its central part) usually has three phases of development: initial phase (before a flare); rapid acceleration phase coinciding with the phase of flare emission growth, and propagation phase with a constant or slowly decreasing velocity [Zhang et al., 2001]. During CME propagation in the corona and interplanetary medium, its structure expands and fills a significant part of the heliosphere. Naturally, the details of CME evolution are determined by the magnetic field structure in the initial configuration, where the flare occurred.

According to modern concepts, a flare and CME are considered as a single process of disturbing the magnetic equilibrium. The highest-speed CME can excite shocks in the corona and interplanetary medium, which can effectively accelerate particles (the width of CME in such events exceeds $100^{\circ}$ ). A sign of the excitation of shocks in the solar corona can be the occurrence of type II radio bursts in a meter range [Reames, 2013]. The composition of the elements, accelerated by shocks in gradual events, largely reflects the content of coronal ions from heights in $(2 \div 3) R_{\odot}$, which are subject to the 


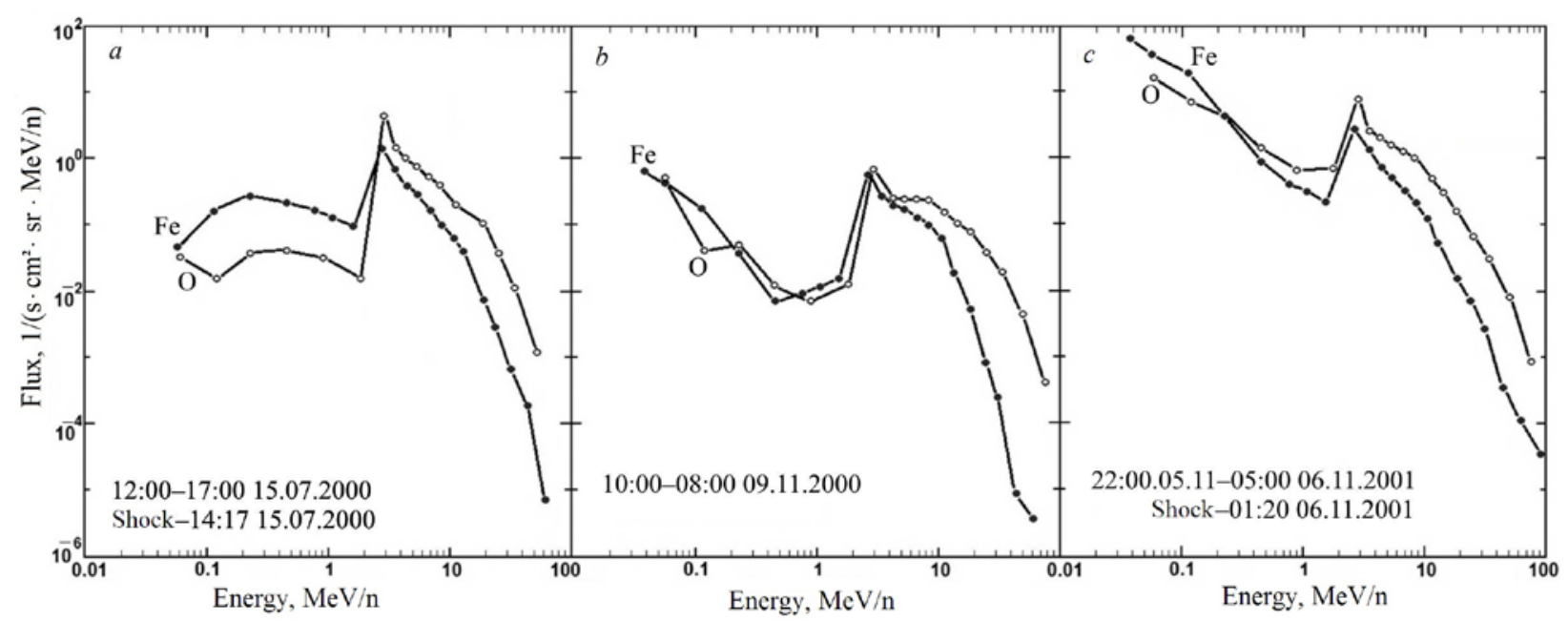

Figure 5. Flux intensity jumps in Fe and O energy spectra in 1.5-2.5 MeV/n

FIP effect [Reames, 2013]. The relative importance of the mechanisms of CME acceleration by shocks depends on the angle between the normal to the wavefront and the magnetic field; the most effective acceleration of ions with energies $\sim \mathrm{MeV}$ is realized near the frontal part of the CME [Kallenrode, 2003]. Furthermore, Cliver et al. [2009] note that a shock can arise in the solar corona during a rapid lateral expansion of the structure of CME when it rises in the corona with possible acceleration of protons up to $\sim 30 \mathrm{MeV}$ (Figure 4, b).

In the magnetic configuration in Figure $4, b$, particles, accelerated in the reconnection region below CME, penetrate into the dense solar atmosphere, causing electromagnetic emission and producing the very flare phenomenon in optical and X-ray ranges. The CME dynamics near the Sun is a significant factor responsible for the intensity of $\sim 10 \mathrm{MeV} / \mathrm{n}$ proton fluxes because during the initial phase CME "launches" a flare in the X-ray range and generates a coronal wave, which initiates the acceleration of protons from the flare region [Torsti et al., 2001]. A part of the accelerated particles is retained in a zone behind the propagating CME and forms the so-called reservoir (magnetic bottle) - an extensive expanding region of plasma in the interplanetary medium with energetic particles and turbulent Alfvén waves [Reames, 2013]. The very process of formation and further evolution of the expanding magnetic reservoir filled with energetic particles can be recorded as a gradual SCR event in the heliosphere. Notice that in the gradual events (Figure 4, b), a small part of the flare-accelerated particles can penetrate into $\mathrm{CME}$ and be further accelerated by a shock; the spectra of the accelerated particles will then have a mixed character. Therefore, $\mathrm{Fe} / \mathrm{O}>1$ plays a special role as a consequence of the FIP effect, since the population of flare-accelerated particles is strongly enriched in Fe. On the contrary, the flux particles accelerated by CME shocks are depleted in Fe $(\mathrm{Fe} / \mathrm{O}<1)$.

Another interesting property is found in the behavior of ion (He, C, O, Fe) energy spectra in particle fluxes from gradual flares. Using GOES data on integral proton fluxes, it has been established that during periods of enhanced fluxes $\left(F_{\mathrm{p}}>10^{2} 1 /\left(\mathrm{s} \cdot \mathrm{cm}^{2} \cdot \mathrm{sr}\right)\right)$ of high-energy protons with energies exceeding $60 \mathrm{MeV}$ there are abrupt changes in ion energy spectra in the form of particle intensity jumps in 1.5-2.5 MeV/n [Minasyants et al., 2016a]. This is typical only of very powerful $\mathrm{X}$ flares accompanied by high-speed CMEs. In 1997-2007, ten such events were registered, with 9 referring to GLE events; and only one, to the class of typical gradual events. The intervals with such enhanced proton fluxes relate to different phases of flare evolution: initial rise, maximum, and a period after 24 hours with the arrival of CME at Earth's orbit, which accompanied the process of appearance and development of a flare. Three events have been recorded in which an additional increase in the intensity of highenergy protons was associated with a CME-driven shock front. Thus, the nature of the intensity jumps in spectra is attributed both to the high-energy particle fluxes, directly accelerated in flares, and to the additional impact of high-speed CME-driven shock fronts. Figure 5 shows Fe and $\mathrm{O}$ energy spectra with flux intensity jumps for the three events.

The intervals in the construction of energy spectra (Figure 5, a, c) are associated with the arrival of CME-driven shock front (GLE events). This Figure shows moments of the shock front arrival; panel $b$, a gradual flare event.

Ion energy spectra in different events exhibit intensity jumps lasting from one hour to one day. The amplitude of these jumps is related to intense fluxes of highenergy protons. When $F_{\mathrm{p}}<10^{2} 1 /\left(\mathrm{s}^{\cdot} \mathrm{cm}^{2} \cdot \mathrm{sr}\right)$, there are no intensity jumps in the energy spectra for protons with $E_{\mathrm{k}}>60 \mathrm{MeV}$. All the intervals in which such jumps occurred are associated with the disturbed phase of flareinduced fluxes.

A distinctive difference in the energy spectra of ion admixtures with intensity jumps is a marked enrichment in Fe ions relative to $\mathrm{O}$ in an energy range $0.06-1.5 \mathrm{MeV} / \mathrm{n}$. This yields $\mathrm{Fe} / \mathrm{O} \geq 1$. The FIP effect in the flux of accelerated high-energy flare-induced particles and their additional acceleration by CME-driven shocks for each 
event has certain differences, but at the same time there is a general tendency. A characteristic feature of GLE events is the presence of these intensity jumps in ion energy spectra. The existence of increased values of $\mathrm{Fe} / \mathrm{O}$ during such jumps leads to the fact that GLE events show higher Fe/O values in $0.06-1.5 \mathrm{MeV} / \mathrm{n}$ than powerful gradual phenomena.

\subsection{RESULTS AND DISCUSSION}

The disturbed active phase of flare-induced fluxes is the most informative in studying properties of accelerated particles and their changes when the particle fluxes move in interplanetary space. Energy spectra with an integration time of $1 \mathrm{hr}$ allow us to trace the properties of particle fluxes of different energies and to find the boundary of the disturbed part of a flare-induced flux, after which the particle fluxes gradually decrease to background values. Processing of energy spectra when integrated over the entire flare-induced flux leads to a significant underestimation of the $\mathrm{Fe} / \mathrm{O}$ values, especially for low ion energy $<2 \mathrm{MeV} / \mathrm{n}$.

We have found abrupt changes in the flux intensity in a range 1.5-2.5 MeV/n during the disturbed phase. Sources of this phenomenon should presumably be considered the presence of high-energy proton fluxes $E_{\mathrm{k}}>60 \mathrm{MeV}$ with $F_{\mathrm{p}}>10^{2} 1 /\left(\mathrm{s} \cdot \mathrm{cm}^{2} \cdot \mathrm{sr}\right)$.

The $\mathrm{Fe} / \mathrm{O}$ distributions in gradual and impulsive events shown in Figure 3 as well as the analysis of existing model concepts of the nature of impulsive and gradual phenomena allow us to draw the following conclusions.

The FIP effect (maximum Fe/O values) is the most pronounced in impulsive flares for $<2 \mathrm{MeV} / \mathrm{n}$ ions (Figure 3). This may be due to the initial accumulation of elements with low FIP (e.g., Fe) in active regions, followed by their release as a result of exchange reconnection with open field lines of adjacent $\mathrm{CHs}$ and escape of energetic particles directly into the interplanetary medium [Wang et al., 2006]. In ion energy spectra with higher energies, the FIP effect weakens and $\mathrm{Fe} / \mathrm{O}$ decreases rapidly. The maximum Fe/O in $0.2-5 \mathrm{MeV} / \mathrm{n}$ (Figure 3) may be caused by acceleration of ions in plasma ejections directed along open field lines from the magnetic reconnection region of the flare. The high efficiency of acceleration of both electrons and ions in simulating the process of magnetic reconnection in current sheets has been confirmed by laboratory experiments with plasma generators [Tomozov, Strokin, 2015].

In gradual events, $\mathrm{Fe} / \mathrm{O}$ smoothly decreases with ion energy with a slight increase in a high-energy region $>30 \mathrm{MeV} / \mathrm{n}$ (Figure 3); it is highest at relatively low energies. In such events, the role of particle acceleration by CME-driven shocks seems to be crucial because shocks can also appear in the corona during accelerated expansion of CME in its ascent phase (Figure 4, b) [Cliver et al., 2009]. As in the previous work [Minasyants et al., 2016b], we note that the Fe/O behavior in spectra of flare-induced fluxes in the interplanetary medium is explained by features of Fe and $\mathrm{O}$ scattering due to their interaction with Alfvén waves, which are generated by energetic protons accelerated by CME shocks; this is consistent with the conclusions drawn by Reames [2014]. These conclusions are also supported by the high correlation found between the proton flux in 5-200 MeV and CME velocity. The correlation is also high in the region of relatively low energies $<20 \mathrm{MeV}$ [Dierckxsens et al., 2015].

When comparing $\mathrm{Fe} / \mathrm{O}$ values obtained for the disturbed phase of particle fluxes in 16 GLE and 16 powerful gradual events in the same energy intervals, notable energy-dependent differences have been found in $\mathrm{Fe} / \mathrm{O}$ values and behavior. This indicates the possibility of separating GLE events into a subclass in the general class of gradual events.

Emphasize that since the FIP effect directly manifests itself in the composition of energetic particles accelerated both by flares and by their related CME, it can play an important diagnostic role in the occurrence and development of solar processes.

\section{BEHAVIOR OF Fe/O IN CORONAL MASS EJECTIONS}

\subsection{Variations in $\mathrm{Fe} / \mathrm{O}$ with ion energy in structures of magnetic clouds during the de- velopment of powerful geomagnetic storms}

In the course of evolution, in solar active regions the free magnetic energy constantly increases, which, when dissipation regions (current sheets) appear, generally gives rise to fast processes of energy release - solar flares and CME. In a current sheet, plasma intensely heats up and particles accelerate up to high energies. Besides, energetic solar flares are almost always accompanied by a fast CME with the formation of shocks, on fronts of which particles effectively accelerate when flare-induced plasma flows move near the Sun and in the interplanetary medium.

When CME propagates in the corona and interplanetary medium, its magnetic structure expands following the shock. In quantitative terms, about half of all CME events that have certain specific characteristics represents magnetic clouds (MC). Direct observations of MC from spacecraft in the interplanetary medium have shown that such structures have a magnetic field that is stronger than that in the surrounding SW, lower proton density $N_{\mathrm{p}}$ and temperature $T_{\mathrm{p}}$, and hence the predominance of magnetic pressure over the gas one.

The most geoeffective CME events are high-speed ejections with bow shocks. They belong to most reliable zone 4 (according to SOHO classification). When CME's structural composition includes MC, the probability of a magnetic storm increases sharply due to the interaction between CME and Earth's magnetosphere.

It has been established that the orientation of the magnetic field in MC at the appearance of CME in a solar active region is similar to that of the field in the sunspot group of the "parent" active region. It was noted that the orientation of the magnetic field in MC is largely preserved when they move in interplanetary space [van Driel-Gesztelyi et al., 2009]. It was also pointed out that 
magnetic clouds with similar field orientations manifest themselves in various ways during the development of geomagnetic disturbances during equinoxes and solstices [Barkhatov et al., 2014].

Observations indicate the existence of smooth rotation of the magnetic field in MC. It is known that when MC appears in the vicinity of Earth, the probability of occurrence of a geomagnetic storm increases due to the interaction between CME and Earth's magnetosphere. MC can generate strong disturbances in SW and have high geoeffectiveness due to the presence of the IMF southward component $B_{z}$ [Gosling et al., 1999; Gonzales et al., 1999; Yermolaev et al., 2009]. There is a turbulent compression region (or shell) between CME bow shock and MC body, which is typified by strong variations in IMF components, by the presence of the $B_{z}$ component, and also by higher plasma density, velocity, and temperature [Yermolaev et al., 2009]. Figure 6 is the schematic of the CME structure including shock front, shell, and MC [Richardson et al., 2010].

Earlier studies carried out by different authors on properties of MC and their role in the development of geomagnetic storms of different power has led to the following conclusions.

It has been established that the magnetic field vector spirals in the MC configuration. Consideration of a change in the MC magnetic field orientation relative to the plane of the ecliptic within a year makes it possible to improve the accuracy of the forecast of geomagnetic storm intensity [Barkhatov et al., 2014]. Shocks and shells are the main sources of geomagnetic disturbances, the further development of which is provided by MC plasma [Barkhatov et al., 2015].

The number of CME events shows a good correlation with Wolf numbers. At the same time, the number of MCs within CME anti-correlates with them. Values of magnetic field and plasma parameters in CME, which in the interplanetary medium are classed as $\mathrm{MC}$, are markedly higher than those in usual CME [Yutian Chi et al., 2015].

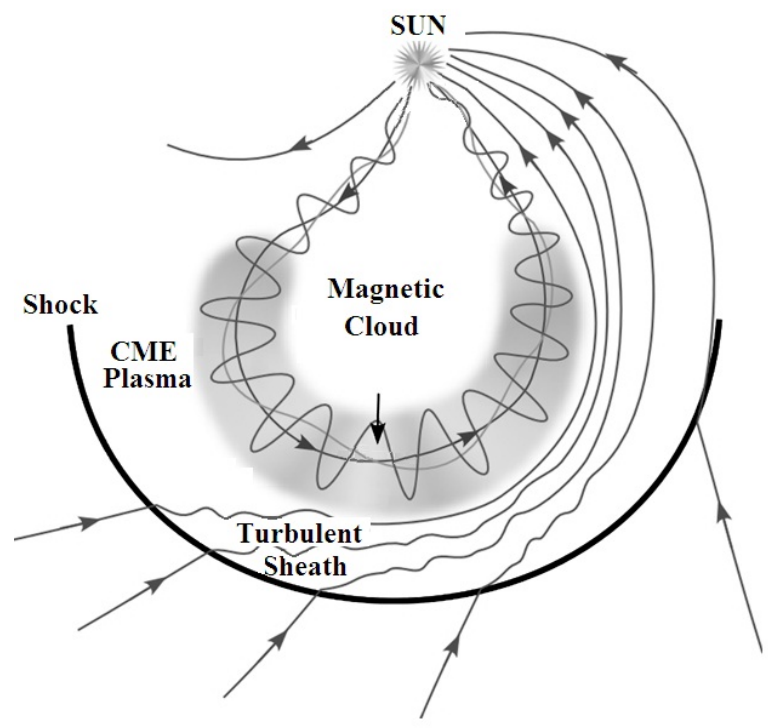

Figure 6. CME structure
The main purpose of further research was to study the $\mathrm{Fe} / \mathrm{O}$ behavior in individual CME structures during the development of geomagnetic storms. We have considered 9 powerful geomagnetic storms with $D s t_{\min }<-200 \mathrm{nT}$. which occurred in 1998-2005 and were related with CME events, including shock fronts (Shock 4), shells, and MC. According to the classification of shock fronts used for SOHO observations, Shock 4 belongs to the highest class and has the largest jumps of $V, N_{\mathrm{p}}, B, T$ in $\mathrm{CME}$ bow shock. The data describing time limits of each of the CME structures have been taken from the Wind ICME Catalogue [https://wind.nasa.gov/fullcatalogue.php].

The method of studying physical conditions for each event also involves comparing time variations of $12 \mathrm{SW}$ parameters in CME structures near Earth's orbit with a time resolution of $5 \mathrm{~min}$ and hourly values of the equatorial geomagnetic index $D s t_{\min }$.

Separately for plasma compression region and for $\mathrm{MC}$ we have constructed the $\mathrm{Fe}$ and $\mathrm{O}$ ion energy spectra in the seven energy ranges. Average values in the intervals, as well as sources of observations and the method of their processing, have been described in Introduction. When calculating the ion energy spectra, we take into account the transit time of each of the CME structures in SW streams. From Fe and $\mathrm{O}$ fluxes, we estimate their abundance in the seven energy ranges for the CME structures. In previous papers, Minasyants et al. [2016b, c] have shown that $\mathrm{Fe} / \mathrm{O}$ in different ion energy ranges is a good indicator of the physical state of a medium under study.

Figure 7 shows the distribution of physical parameters in the CME structure and the course of the development of magnetic storm according to the Dst index for the November 9-10, 2004 event; Figure 8 illustrates $\mathrm{Fe}$ and $\mathrm{O}$ energy spectra in CME plasma for the same event.

Notice that in calculating the ion energy spectra in the compression region, we account for the effect of the CME bow shock front.

Tables 4 and 5 list the calculated Fe/O values for the seven energy ranges in the shell and MC in the structure of the nine CMEs during the development of strong geomagnetic storms with $D s t_{\min }<-200 \mathrm{nT}$.

Let us analyze the results of processing of data (Figures 6-8 and Tables 4, 5) on the behavior of $\mathrm{Fe} / \mathrm{O}$ in MC structures.

We add that the ratio of admixture $\mathrm{Fe} / \mathrm{O}$ (as well as $\mathrm{Mg} / \mathrm{O}$, etc.) is a measure of the FIP effect in the solar atmosphere, which has been described in sufficient detail in Introduction.

Consequently, the $\mathrm{Fe} / \mathrm{O}$ ratio in different coronal structures and SW may have different values and serve as an indicator of physical processes in solar plasma (e.g., in coronal plasma, the mean value $\left.(\mathrm{Fe} / \mathrm{O})_{\mathrm{cor}} /(\mathrm{Fe} / \mathrm{O})_{\mathrm{ph}} \sim 4\right)$. The analysis of 54 SEP events (November 1994 - June 2012) in an energy range $2-15 \mathrm{MeV} / \mathrm{n}$ allowed a conclusion to be drawn [Reames, 2014] that the main cause of variations in the abundance of heavy ions in the interplanetary medium (and hence $\mathrm{Fe} / \mathrm{O}$ variations) is their scattering. The scattering is by Alfvén waves and depends on the magnetic rigidity of particles. This process 

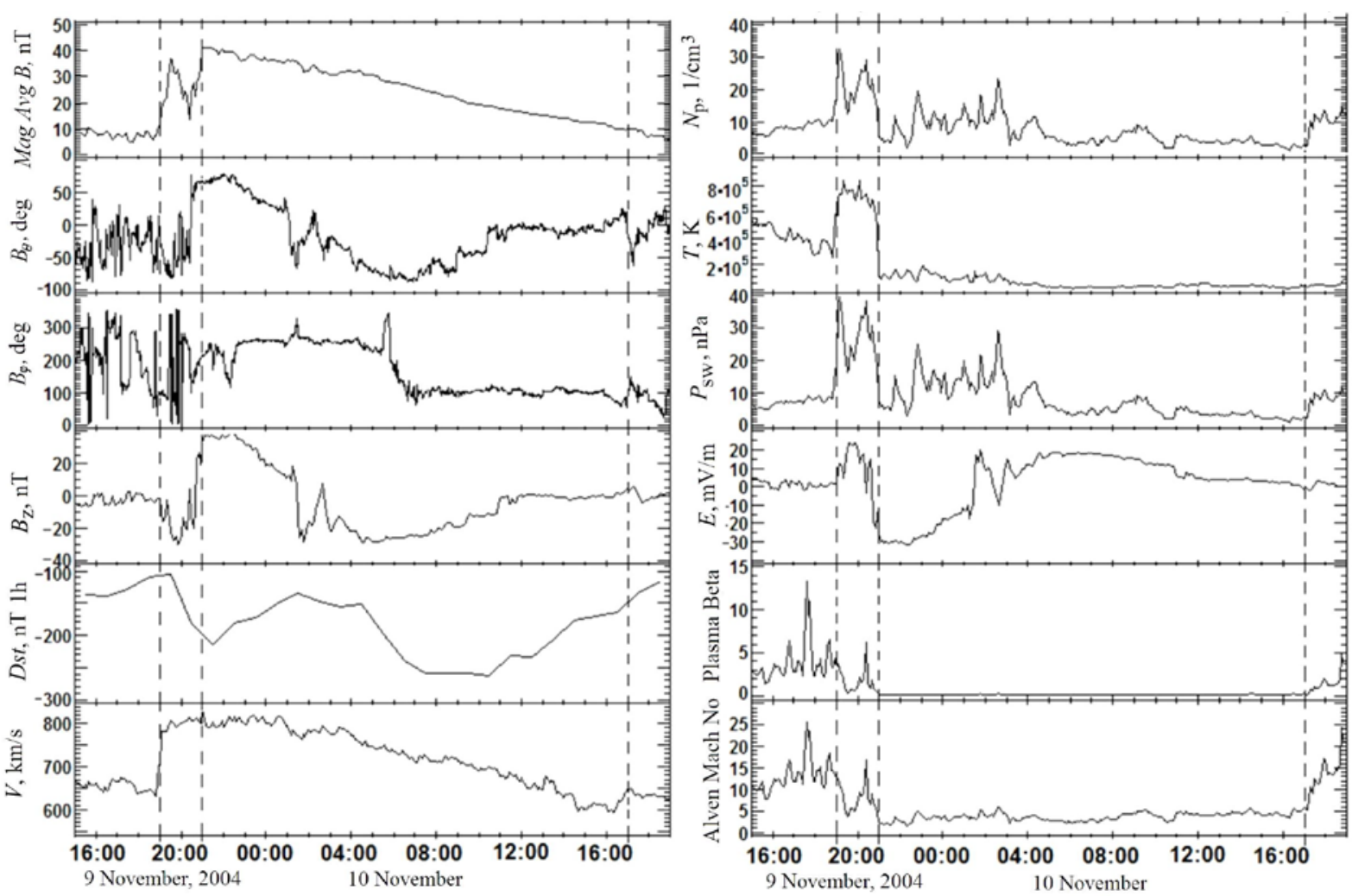

Figure 7. Time variations in SW plasma parameters on November 9-10, 2004. Vertical dashed lines from left to right show: the moment of the arrival of a CME-driven shock (UV), followed by a shell; between the second and third dashed lines is MC

Table 4

Calculated $\mathrm{Fe} / \mathrm{O}$ ratios in the shell region

for 9 CME in 7 ranges of ion energy spectrum

\begin{tabular}{|c|c|c|c|c|c|c|c|}
\hline \multirow{2}{*}{ Shells } & \multicolumn{7}{|c|}{ Fe/O } \\
\cline { 2 - 8 } & $0.06 \mathrm{MeV} / \mathrm{n}$ & $0.23 \mathrm{MeV} / \mathrm{n}$ & $1.81 \mathrm{MeV} / \mathrm{n}$ & $5.30 \mathrm{MeV} / \mathrm{n}$ & $13.00 \mathrm{MeV} / \mathrm{n}$ & $30.90 \mathrm{MeV} / \mathrm{n}$ & $75.69 \mathrm{MeV} / \mathrm{n}$ \\
\hline 02:23.25.09-04:32.25.09.1998 & 0.468 & 0.194 & 0.134 & 0.107 & 0.078 & - & - \\
\hline 14:23.15.07-21:04.15.07.2000 & 0.562 & 0.657 & 0.882 & 0.275 & 0.057 & 0.028 & 0.675 \\
\hline 18:09.11.08-06:04.12.08.2000 & 0.566 & 0.189 & 0.071 & 0.002 & 0.166 & 0.870 & 0.415 \\
\hline 05:51.24.11-15:46.24.11.2001 & 0.630 & 0.365 & 0.294 & 0.250 & 0.117 & 0.059 & 0.282 \\
\hline 06:00.29.10-11:15.29.10.2003 & 0.610 & 0.451 & 0.320 & 0.202 & 0.040 & 0.004 & 0.270 \\
\hline 08:35.20.11-11:30.20.11.2003 & 0.245 & 0.170 & 0.178 & 0.152 & 0.181 & 0.207 & 0.117 \\
\hline 17:58.07.11-22:45.07.11.2004 & 0.242 & 0.090 & 0.346 & 0.129 & 0.101 & 0.095 & 0.164 \\
\hline 18:25.09.11-20:37.09.11.2004 & 0.267 & 0.186 & 0.156 & 0.079 & 0.043 & 0.059 & 0.436 \\
\hline $02: 10.15 .05-04: 46.15 .05 .2005$ & 0.255 & 0.141 & 0.127 & - & - & - & - \\
\hline Mean values & $0.427 \pm 0.057$ & $0.273 \pm 0.061$ & $0.279 \pm 0.082$ & $0.149 \pm 0.032$ & $0.140 \pm 0.036$ & $0.189 \pm 0.116$ & $0.337 \pm 0.072$ \\
\hline
\end{tabular}

Table 5

Calculated values of the $\mathrm{Fe} / \mathrm{O}$ ratio in $\mathrm{MC}$ for 9 CMEs in 7 ranges of ion energy spectrum

\begin{tabular}{|c|c|c|c|c|c|c|c|}
\hline \multirow{2}{*}{ Magnetic cloud } & \multicolumn{7}{|c|}{$\mathrm{Fe} / \mathrm{O}$} \\
\cline { 2 - 8 } & $0.06 \mathrm{MeV} / \mathrm{n}$ & $0.23 \mathrm{MeV} / \mathrm{n}$ & $1.81 \mathrm{MeV} / \mathrm{n}$ & $5.30 \mathrm{MeV} / \mathrm{n}$ & $13.00 \mathrm{MeV} / \mathrm{n}$ & $30.90 \mathrm{MeV} / \mathrm{n}$ & $75.69 \mathrm{MeV} / \mathrm{n}$ \\
\hline 04:33.25.09-17:00.26.09.1998 & 0.438 & 0.313 & 0.504 & 0.212 & 0.233 & 0.343 & 0.080 \\
\hline 21:05.15.07-13:28.16.07.2000 & 0.507 & 0.291 & 0.253 & 0.102 & 0.061 & 0.029 & 0.173 \\
\hline 06:05.12.08-05:06.13.08.2000 & 0.338 & 0.636 & 0.780 & 1.046 & 0.308 & 0.282 & 0.233 \\
\hline 15:47.24.11-13:17.25.11.2001 & 0.561 & 0.360 & 0.281 & 0.209 & 0.143 & 0.082 & 0.064 \\
\hline 11:16.29.10-01:55.30.10.2003 & 0.443 & 0.280 & 0.197 & 0.120 & 0.031 & 0.074 & 0.173 \\
\hline 11:31.20.11-01:40.21.11.2003 & 0.963 & 0.475 & 0.403 & 0.177 & 0.166 & 0.158 & 0.073 \\
\hline 22:46.07.11-09:21.09.11.2004 & 0.532 & 0.264 & 0.187 & 0.069 & 0.047 & 0.044 & 0.280 \\
\hline 20:38.09.11-16:47.10.11.2004 & 0.561 & 1.509 & 0.312 & 0.112 & 0.057 & 0.071 & 0.243 \\
\hline $04: 47.15 .05-22: 47.16 .05 .2005$ & 0.291 & 0.132 & 0.083 & 0.011 & 0.008 & 0.209 & 0.132 \\
\hline Mean values & $0.515 \pm 0.064$ & $0.473 \pm 0.138$ & $0.333 \pm 0.069$ & $0.229 \pm 0.104$ & $0.117 \pm 0.034$ & $0.144 \pm 0.037$ & $0.161 \pm 0.027$ \\
\hline
\end{tabular}




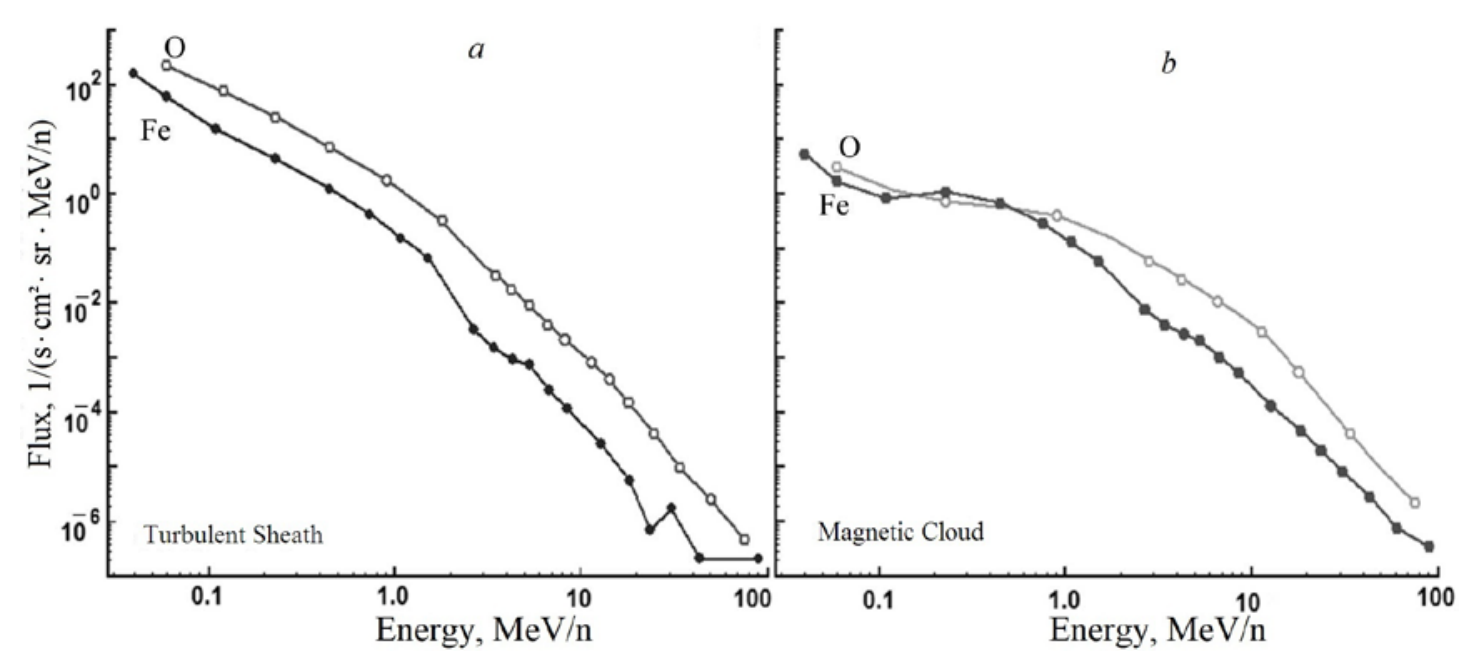

Figure 8. Fe and O energy spectra for shell (a) and magnetic cloud (b) in CME plasma on November 9-10, 2004

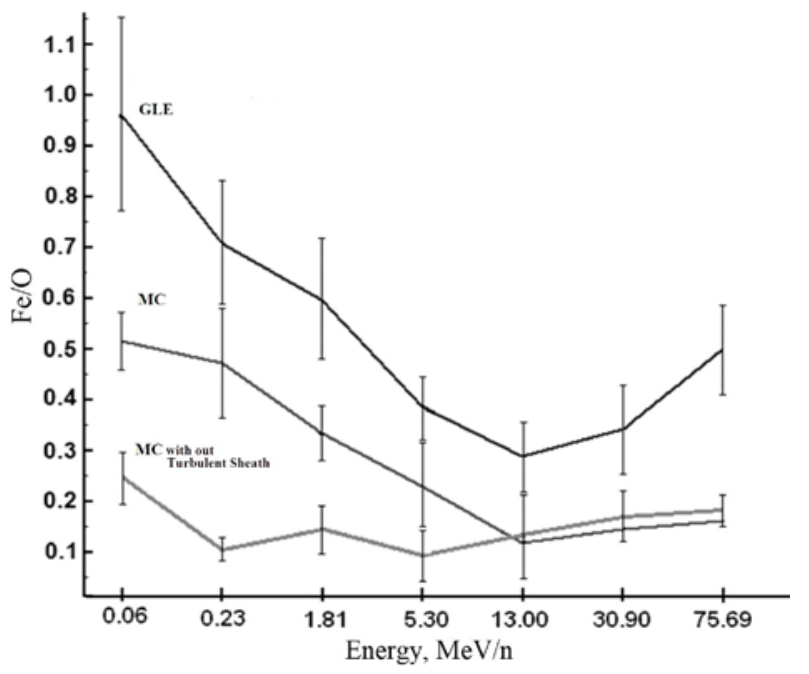

Figure 9. Variations in mean $\mathrm{Fe} / \mathrm{O}$ with ion energy in various manifestations of solar activity: in GLE particle fluxes, in MC. The lower curve is MC without CME-driven shock front and shell

occurs during particle propagation from the region of their acceleration by CME-driven shocks. In the interplanetary medium, Alfvén waves are excited by energetic protons and ions, which are accelerated by both flares and CMEdriven shocks [Reames, 2014]. The Fe ions are scattered by Alfvén waves less effectively than the $\mathrm{O}$ ions, therefore, when propagating in a turbulent medium, the Fe ions can outrun the $\mathrm{O}$ ions. This yields $\mathrm{Fe} / \mathrm{O}>1$.

If flare and CME generating regions are spatially close or bound by a magnetic field, some of the particles accelerated in the flare can be captured by CME and receive additional acceleration in a shock during propagation in the interplanetary medium [Tomozov, 2012].

Analyzing the $\mathrm{Fe} / \mathrm{O}$ behavior in the structure of MC (Figure $8 a, b$ ) causing the November 9-10, 2004 geomagnetic storm (Figure 7), we can note the following points. First, $\mathrm{Fe} / \mathrm{O}<1$ in the MC shell (Figure $8, a$ ) behind the shock in an energy range from 0.01 to $100 \mathrm{MeV} / \mathrm{n}$. This indicates a weak influence of Alfvén turbulence on ion scattering, including $\mathrm{O}$ ions with high FIP. We should emphasize that the Alfvén wave turbulence also exists behind the shock. Second, within the MC (Figure 8, b) there is a small area with $\mathrm{Fe} / \mathrm{O}>1$ in $0.2-0.6 \mathrm{MeV} / \mathrm{n}$. This may be associated either with the influence of scattering by Alfvén waves or with the effect of preferential accumulation of ions with low FIP in the magnetic structure in front of CME.

Figure 9 shows variations in mean $\mathrm{Fe} / \mathrm{O}$ values in the seven ion energy ranges for $\mathrm{MC}$ in structures of nine CMEs, which caused strong geomagnetic storms with $D s t_{\min }<-200 \mathrm{nT}$. By comparison, we show the Fe/O behavior for GLE events. It can be noted (Figure 8) that in the entire ion energy range $\mathrm{Fe} / \mathrm{O}<1$ both in $\mathrm{MC}$ and in the shell behind the shock wave. At the same time, a certain $\mathrm{Fe} / \mathrm{O}$ peak is observed in the shell for $1.81 \mathrm{MeV} / \mathrm{n}$, similar to that in impulsive flare events with the same energy. Note also that there is a certain break of the Fe/O dependence in MC within 0.2-0.6 MeV/n, i.e., just as in the November 9-10, 2004 phenomenon, Fe/O $>1$ (Figure 8, b).

We have also found MC structures that had no bow shocks (i.e., no shells in front of MC) and caused only moderate short-term geomagnetic storms with $D s t_{\min }>-50 \mathrm{nT}$. The averaged curve of the Fe/O variation, plotted from $\mathrm{O}$ and $\mathrm{Fe}$ energy spectra for four MCs with the said properties, is also given in Figure 9. A distinctive feature of this curve is rather low values of $\mathrm{Fe} / \mathrm{O}$ as compared to the geoeffective $\mathrm{MC}$ in the energy range $E_{\mathrm{k}}<13 \mathrm{MeV} / \mathrm{n}$ and slightly increased $\mathrm{Fe} / \mathrm{O}$ at higher ion energies. This fact also underlines the prognostic significance of the FIP effect for determining geoeffectiveness of solar events.

Magnetic clouds with shells, which are sources of strong geomagnetic storms, have higher abundance of Fe ions in SW streams, comparable to its abundance in particle fluxes from gradual flares. The analysis of Fe and $\mathrm{O}$ energy spectra in fluxes of nine MCs, which caused strong geomagnetic storms, has shown that the $\mathrm{Fe} / \mathrm{O}$ index is an indicator of the physical state of the medium under study. In the shell throughout the ion energy range, $\mathrm{Fe} / \mathrm{O}<<1$. This indicates a low efficiency of scattering of $\mathrm{O}$ ions with high FIP by Alfvén turbulence. In MC within 0.2-0.6 MeV/n range, Fe/O 1. This 
Time of passage of CME structural elements, electric field E, degree of ionization of iron ions $\mathrm{Q}$, and $\mathrm{Fe} / \mathrm{O}$ ratio in $\mathrm{CME}$ plasma

\begin{tabular}{|r|c|c|c|c|c|c|}
\hline No. & $\begin{array}{c}\text { Arrival of CME shock } \\
\text { front }\end{array}$ & $\begin{array}{c}\text { Shell end/ } \\
\text { Frontal part of CME } \\
\text { body }\end{array}$ & CME end & $E, \mathrm{mV} / \mathrm{m}$ & $Q(\mathrm{Fe})$ & $\mathrm{Fe} / \mathrm{O}$ \\
\hline 1 & $04: 20.08 .11 .1998$ & $08: 00.08 .11$. & $23: 31.08 .11$. & $7.09 \pm 0.16$ & $13.18 \pm 0.18$ & $0.37 \pm 0.05$ \\
\hline 2 & $14: 17.15 .07 .2000$ & $19: 10.15 .07$. & $14: 30.16 .07$. & $21.61 \pm 6.89$ & $16.80 \pm 0.18$ & $0.81 \pm 0.17$ \\
\hline 3 & $18: 19.11 .08 .2000$ & 06.05 .12 .08$. & $05: 05.13 .08$. & $14.55 \pm 1.19$ & $14.92 \pm 0.17$ & $0.63 \pm 0.04$ \\
\hline 4 & $00: 14.31 .03 .2001$ & $12: 00.31 .03$. & $02: 00.02 .04$. & $15.47 \pm 2.14$ & $15.58 \pm 0.19$ & $0.79 \pm 0.13$ \\
\hline 5 & $23: 57.17 .04 .2001$ & $06: 20.18 .04$. & $07: 20.19 .04$. & $4.28 \pm 1.53$ & $10.35 \pm 0.06$ & $0.23 \pm 0.02$ \\
\hline 6 & $04: 28.28 .04 .2001$ & $17: 59.28 .04$. & $04: 48.02 .05$. & - & $15.99 \pm 0.11$ & $0.88 \pm 0.10$ \\
\hline 7 & $02: 33.28 .10 .2001$ & $11: 00.28 .10$. & $03: 17.30 .10$. & $5.92 \pm 0.43$ & $14.36 \pm 0.89$ & $0.40 \pm 0.05$ \\
\hline 8 & $05: 51.24 .11 .2001$ & $15: 47.24 .11$. & $13: 17.25 .11$. & $5.38 \pm 3.45$ & $13.26 \pm 0.32$ & $0.53 \pm 0.06$ \\
\hline 9 & $04: 08.23 .04 .2002$ & $07: 11.24 .04$. & $23: 59.24 .04$ & $0.44 \pm 0.07$ & $11.37 \pm 0.07$ & $0.18 \pm 0.01$ \\
\hline 10 & $10: 20.26 .08 .2002$ & $14: 23,26.08$. & $13: 40.27 .08$. & $2.46 \pm 0.41$ & $9.41 \pm 0.07$ & $0.27 \pm 0.01$ \\
\hline 11 & $05: 51.29 .10 .2003$ & $11: 16.29 .10$. & $10: 00.30 .10$. & - & $16.31 \pm 0.25$ & $0.79 \pm 0.15$ \\
\hline 12 & $16: 00.30 .10 .2003$ & $04: 30.31 .10$. & $23: 00.02 .11$. & - & $16.77 \pm 0.20$ & $1.20 \pm 0.23$ \\
\hline 13 & $05: 53.04 .11 .2003$ & $14: 00.04 .11$. & $12: 00.06 .11$. & $0.87 \pm 0.05$ & $9.74 \pm 0.07$ & $0.16 \pm 0.01$ \\
\hline 14 & $18: 24.09 .11 .2004$ & $20: 38.09 .11$. & $16: 47.10 .11$. & $15.80 \pm 0.85$ & $13.87 \pm 0.18$ & $0.64 \pm 0.08$ \\
\hline 15 & $16: 48.21 .01 .2005$ & $23: 45.21 .01$. & $19: 12.22 .01$. & $1.75 \pm 0.60$ & $12.23 \pm 0.28$ & $0.21 \pm 0.07$ \\
\hline 16 & $02: 19.15 .05 .2005$ & $04: 17.15 .05$. & $22: 47.16 .05$. & $22.51 \pm 7.16$ & $16.34 \pm 0.19$ & $0.89 \pm 0.09$ \\
\hline 17 & $13: 57.14 .12 .2006$ & $22: 36.14 .12$. & $13: 40.15 .12$. & $12.43 \pm 0.50$ & $16.31 \pm 0.10$ & $0.51 \pm 0.10$ \\
\hline
\end{tabular}

may be due to the enhancement of scattering by Alfvén waves or to the effect of preferential accumulation of ions with low FIP in MC in front of CME.

\subsection{Influence of ion ionization on the FIP effect in coronal mass ejections}

For the analysis, we have selected $17 \mathrm{CME}$ events (1998-2006), which had a pronounced bow shock front at the highest level of reliability. According to the SOHO classification, they had an index Shock 4. All the CME events were of halo type. As CME propagates in the corona and interplanetary medium, its magnetic structure expands following the shock. The shell, which is located immediately behind the shock front, features strong variations in IMF components $B$, higher proton density $N_{\mathrm{p}}$, velocity $V_{\mathrm{p}}$, and temperature $T_{\mathrm{p}}$ [Yermolaev et al., 2009]. Behind the shell is the CME body. The data describing time limits of each of the CME structures have been taken from the Wind ICME Catalogue [https://wind.nasa.gov/fullcatalogue.php].

As the active process develops, a part of the flare plasma can penetrate into the CME region, i.e. the spectrum of accelerated particles in this zone has a mixed character. In this regard, a special role is played by $\mathrm{Fe} / \mathrm{O}>1$, which results from the FIP effect since the population of flare-accelerated particles is strongly enriched in Fe. Particles accelerated by CME-driven shocks are, on the contrary, depleted in $\mathrm{Fe}(\mathrm{Fe} / \mathrm{O}<1)$.

We have used ACE/SWICS data averaged over $1 \mathrm{hr}$, which are only for one energy range $E_{\mathrm{k}}=0.5 \div 100 \mathrm{keV} / \mathrm{e}$. In addition to $\mathrm{Fe} / \mathrm{O}$ values, we employed indices characterizing the degree of $\mathrm{Fe}$ and $\mathrm{O}$ ionization, $\mathrm{Q}(\mathrm{Fe}), Q(\mathrm{O})$. To assess quantitatively $Q(\mathrm{Fe})$ and $\mathrm{Fe} / \mathrm{O}$, we calculated their mean values in a 6-hour interval during an increase in time profiles of these parameters (Table 6).

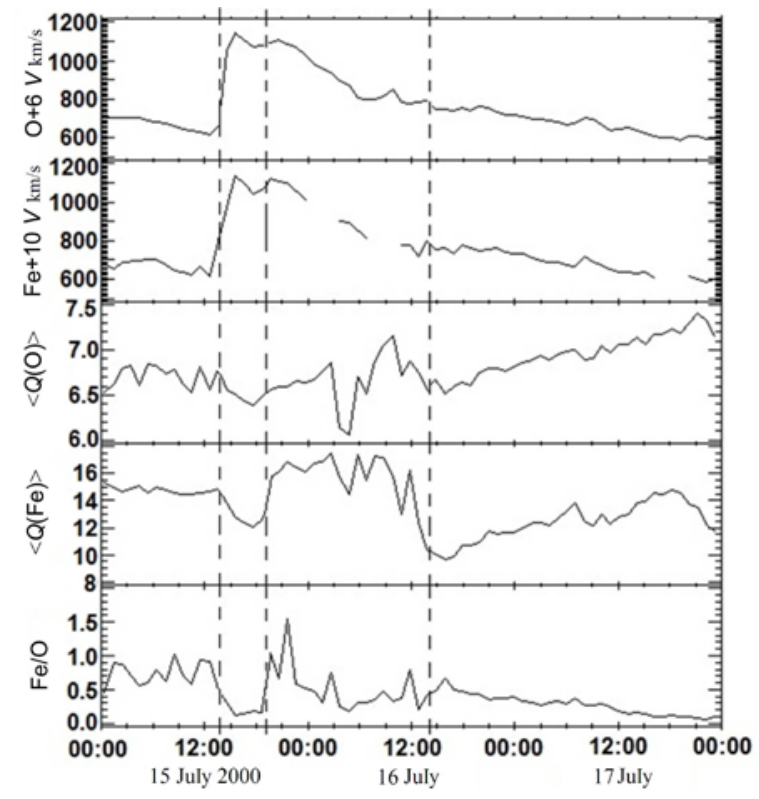

Figure 10. Time variations in physical parameters in the July 15-16, 2000 CME structure

Figures 10 and 11 show time variations in the velocity of $\mathrm{O}$ and Fe ions, their degree of ionization $Q(\mathrm{O})$, $\mathrm{Q}(\mathrm{Fe})$, and $\mathrm{Fe} / \mathrm{O}$ for $\mathrm{CME}$ arising from the development of strong GLE events. Vertical dashed lines from left to right indicate the moment of arrival of a CME-driven shock front (UV), shell, and CME body (between the second and third dashed lines).

Note that the FIP effect associated with an increase in the abundance of Fe ions (low FIP) with respect to $\mathrm{O}$ ions (high FIP) is enhanced when there are higher charge state ions in CME plasma $Q(\mathrm{Fe})$ (July 15-16, 2000). The August 26-27, $2002 \mathrm{CME}$, on the contrary, exhibits low, almost background, values of $Q(\mathrm{Fe})$, and accordingly Fe/O 


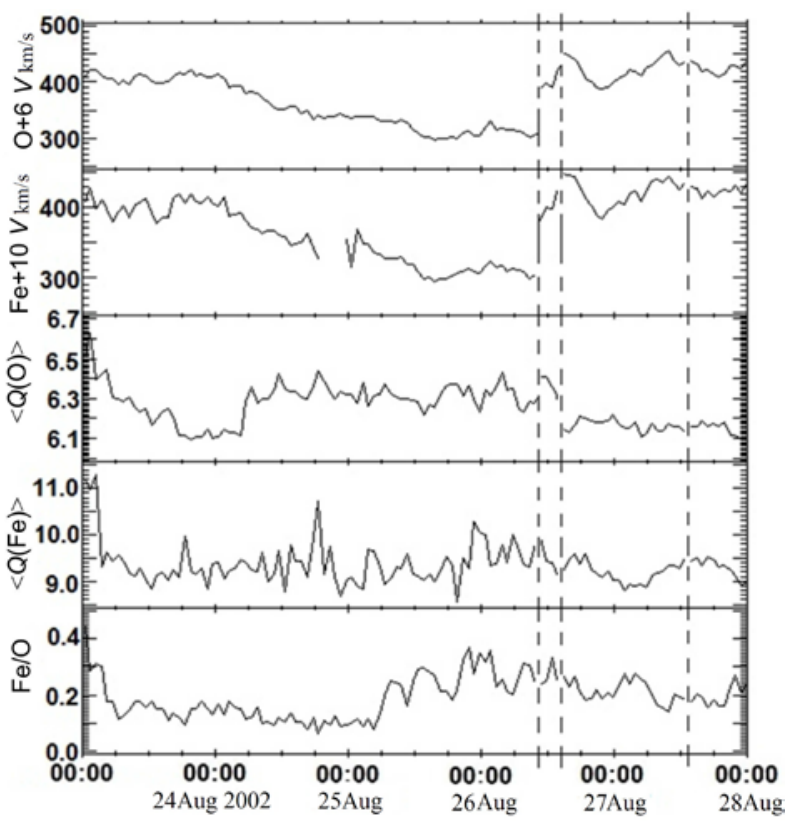

Figure 11. Time variations of physical parameters in the August 26-27, 2002 CME structure

do not show such enhancement. This probably indicates that there is not more energetic flare plasma in the CME structure.

The consideration of time variations in parameters for all the selected CME events indicates their individual properties associated with the composition of CME plasma, which manifest themselves in different values of $Q(\mathrm{Fe}), Q(\mathrm{O})$ as well as in $\mathrm{Fe} / \mathrm{O}$. To assess quantitatively the $Q(\mathrm{Fe}), \mathrm{Fe} / \mathrm{O}$ parameters in each $\mathrm{CME}$, we calculated their mean values in the 6-hour interval during an increase in time profiles of these indices. Another feature was found in the CME structure. The shell characterized by high values of $V, N_{\mathrm{p}}, B, T$ associated with a CME-driven shock generally does not show an increase in $Q(\mathrm{Fe}), Q(\mathrm{O}), \mathrm{Fe} / \mathrm{O}$. This suggests a low efficiency of ion scattering by Alfvén turbulence in this region (the turbulence of Alfvén waves also exists behind the shock).

Table 6 shows the time of passage of all structural elements of the CME events under study: shock front, shell, and CME body. For plasma of the CME events, some columns present electric field strength $E$, charge states of $\mathrm{Fe}$ ions $Q(\mathrm{Fe})$, and $\mathrm{Fe} / \mathrm{O}$ ratio, with averaging errors.

Note that the average values of $\mathrm{Fe} / \mathrm{O}$ in ion energy spectra $E_{\mathrm{k}}=0.06 \mathrm{MeV} / \mathrm{n}$ for MC (Table 5) and in CME structure (Table 6) for $E_{\mathrm{k}}=0.5 \div 100 \mathrm{keV} / \mathrm{e}$ show good agreement.

$Q(\mathrm{Fe})$ and $\mathrm{Fe} / \mathrm{O}$ in $\mathrm{CME}$ plasma are compared in Figure 12 . With $Q(\mathrm{Fe})<13$ there is practically no change in Fe/O with increasing $Q(\mathrm{Fe})$. But when $Q(\mathrm{Fe}) \geq 13$, the FIP effect is sharply enhanced due to an increase in the abundances of Fe ions. This is likely to be associated with the presence of flare plasma in CME body.

This result agrees with the conclusions drawn by Zurbuchen et al. [2016], who have studied abundances of various elements in the CME structure, revealing a tendency for the FIP effect to increase with $Q(\mathrm{Fe})$.

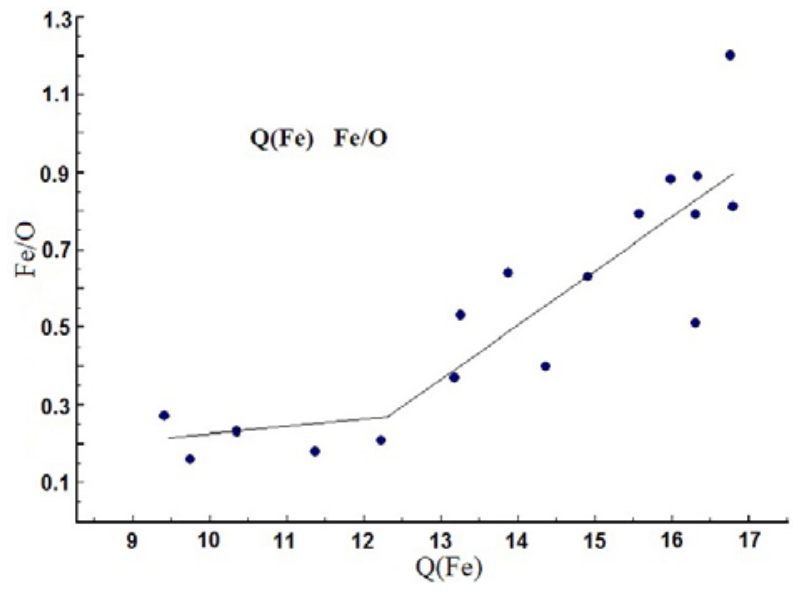

Figure 12. Degree of Fe ionization versus Fe/O values for the CME events considered

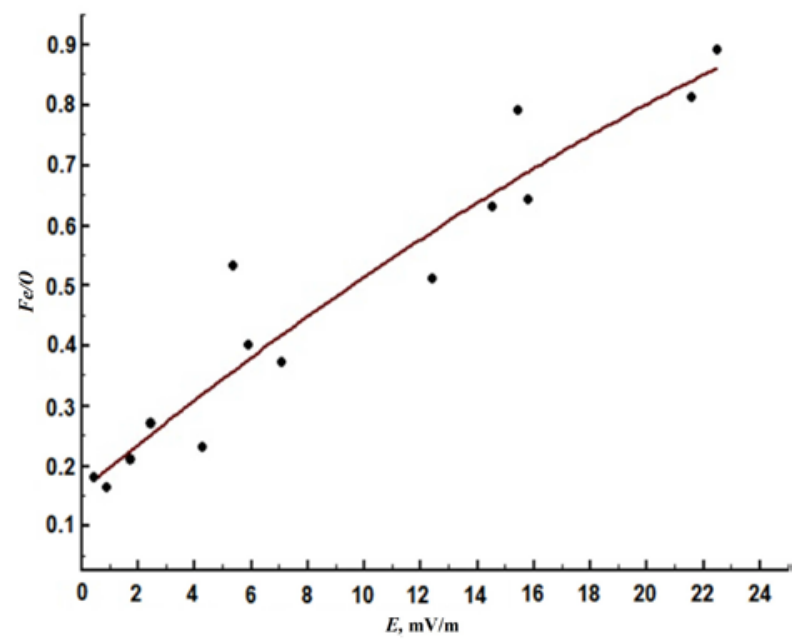

Figure 13. Electric field strength $E \mathrm{mV} / \mathrm{m}$ versus $\mathrm{Fe} / \mathrm{O}$ as derived from CME plasma observations

A special study was devoted to the identification of a correlation between variations in $V, B, N_{\mathrm{p}}, E, T$ and values of $\mathrm{Fe} / \mathrm{O}, Q(\mathrm{Fe})$ in the $\mathrm{CME}$ structure. Of all the parameters considered, $E$ values, which are naturally associated with electron density, show the best correlation with variations in $Q(\mathrm{Fe})$ and $\mathrm{Fe} / \mathrm{O}$ in CME plasma (Figure 13).

Thus, the most probable cause of the increase in $Q(\mathrm{Fe})$ and accordingly in $\mathrm{Fe} / \mathrm{O}$ can be considered to be the penetration of energetic flare plasma, significantly enriched in electrons, into the CME body during the initial phase of its development. The relative contribution of the flare-induced flux can be estimated from a change in the electron density in CME plasma with respect to its background in SW (data from the Wind satellite). If the flare contribution is absent or small, the electron density enhancement $\Delta N_{\mathrm{e}}=0.5 \div 1.0 \mathrm{~cm}^{-3}$; with a significant contribution, $\Delta N_{\mathrm{e}}=5 \div 10 \mathrm{~cm}^{-3}$.

\section{EFFECT OF ANOMALOUS COSMIC RAY COMPONENT ON Fe/O INDEX BEHAVIOR AT SOLAR MINIMUM}

The long-term minimum of solar cycle 23 allowed us to observe variations in fluxes of minor chemical elements 


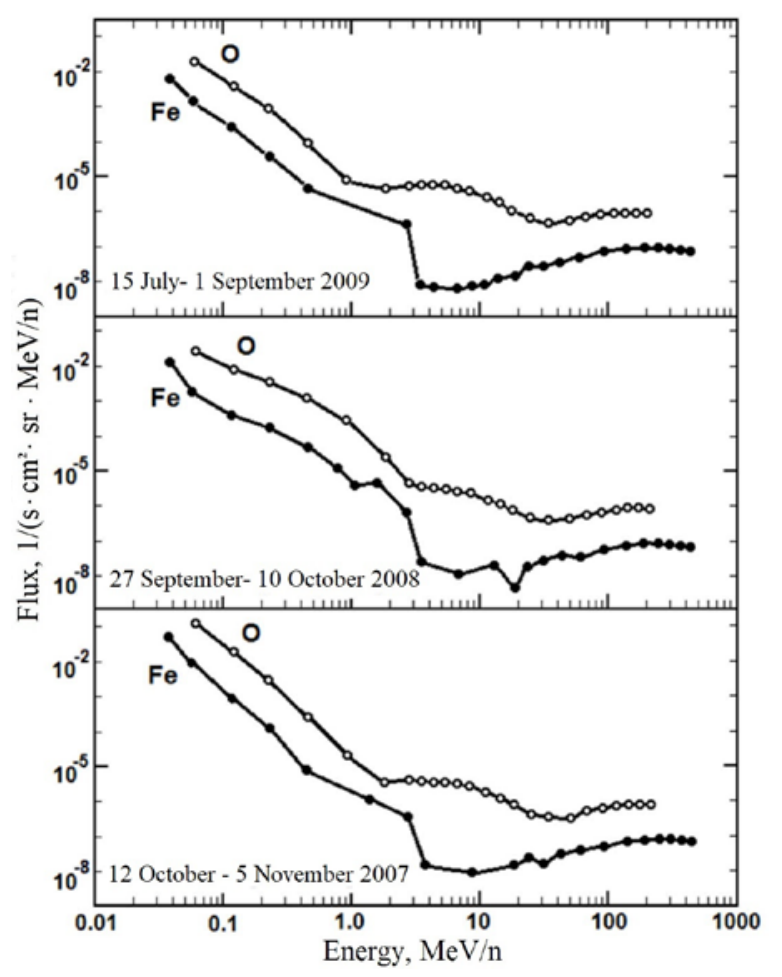

Figure 14. Energy spectra of Fe and O ions of different origin ( $<2 \mathrm{MeV} / \mathrm{n}$ - solar, $>2 \mathrm{MeV} / \mathrm{n}$ - ACR and GCR) in intervals without active regions on the Sun

in SW. Over the history of routine observations of the Sun since 1700 , only cycles 11 and 14 at solar minimum had lower Wolf numbers than cycle 23. December 2008 is defined as a minimum of the previous solar cycle, therefore we have chosen the period from April 1, 2007 to December 31, 2009 to study the solar wind. During this period, for 669 days, there were no active regions on the Sun. In the remaining days there were only pores and sunspots with very small areas, which did not give rise to appreciable disturbances in SW.

The analysis of filtergrams from the space observatory SDO/AIA $171 \AA$, $304 \AA$, and $1700 \AA$ has shown that the main manifestations of solar activity during spotless periods are small areas of increased brightness in the corona in the form of separate clusters of fine plasma jets that rise to a small height. They do not create visible structures in the form of closed loops, similar to those existing in active regions. In the photosphere there are bright plages in the zone of localization of these jets. Almost over the entire solar disk at this time there are bright $\mathrm{X}$-ray points (including those in $\mathrm{CH}$ ) manifestations of small-scale reconnection processes. In the same period, there are extensive dark CHs (sources of fast SW), not only in polar regions, but also in equatorial regions.

For three intervals with complete absence of sunspots in different years of solar minimum, we have constructed energy spectra of $\mathrm{O}$ and Fe ions; the total ion energy range is $0.06-500 \mathrm{MeV} / \mathrm{n}$ (Figure 14). Beginnings of the intervals in the energy spectra are shifted three days ahead to obtain parameters of low solar activity. Furthermore, to the seven energy intervals used earlier in $\mathrm{Fe} / \mathrm{O}$ calculations (see Introduction) we add the eighth one with a mean value of 192.39 MeV/n.
All the studies on properties of particle fluxes of different energies presented below have been carried out by constructing energy spectra of ions, including $\mathrm{Fe}$ and $\mathrm{O}$, and by using them to compute $\mathrm{Fe} / \mathrm{O}$ values, which are indicators of the physical state of the medium under study [Tomozov, 2012].

The structure and properties of the composition of admixtures in SW plasma streams are determined by two main factors: generation of energetic particles on the Sun and conditions of their propagation in the interplanetary medium, as well as the arrival of GCR at the heliosphere. The main causes affecting the change in propagation conditions of a low-energy part of GCR are the number and nature of distribution of magnetic inhomogeneities, as well as magnetic field fluctuations in the heliosphere. The absence of active regions on the Sun leads to a minimization of sources of GCR scattering in the heliosphere and hence to an enhancement of the GCR effect. With increasing $\mathrm{Fe}$ and $\mathrm{O}$ ion energies, $\mathrm{Fe} / \mathrm{O}$ values can consistently describe the main SW characteristics. At $E_{\mathrm{k}}<2 \mathrm{MeV} / \mathrm{n}$, the dominant effect of the Sun is most pronounced; at $E_{\mathrm{k}}=2 \div 30 \mathrm{MeV} / \mathrm{n}$, the effect of ACR; at $E_{\mathrm{k}}>30 \mathrm{MeV} / \mathrm{n}$, the effect of GCR particles [Garrard et al., 1997].

The calculated and presented in Figure $14 \mathrm{Fe}$ and $\mathrm{O}$ energy spectra for SW in the absence of sunspots have some features. Smooth spectra profiles in these cases exhibit anomalous behavior with a rather significant dip for Fe ions in the energy range $\sim 2-30 \mathrm{MeV} / \mathrm{n}$, whereas profiles of $\mathrm{O}$ ion spectra, on the contrary, do not show the decrease - they demonstrate slight enhancement. At $E_{\mathrm{k}}>30 \mathrm{MeV}$ n, instead of the usual decrease there is a steady increase in $\mathrm{O}$ and $\mathrm{Fe}$ fluxes for this energy interval due to the GCR effect.

ACR particles are formed when neutral atoms with high FIP ( $\mathrm{H}, \mathrm{He}, \mathrm{N}, \mathrm{O}$, and $\mathrm{Ne}$ ) penetrate from the interstellar medium into the heliosphere. Then they are ionized due to charge exchange with SW protons or under the impact of solar UV radiation, and are captured by SW [Fisk et al., 1974; Reames, 2001]. When approaching the Sun, they are carried away in the SW stream outside and, when reaching the boundaries of the heliosphere, they are accelerated by the Fermi mechanism to $\sim 10 \mathrm{MeV} / \mathrm{n}$ in the boundary shock. Then they can return to the Sun again. It has been proved that this process can repeat itself many times. Note that ions of elements with low FIP are ionized already in the local interstellar medium and cannot penetrate into the heliosphere. The proposed acceleration mechanism of ACR particles may explain the increase in fluxes of elements with high FIP, including oxygen. At the same time, elements with low FIP should not show this increase - this refers to the C, $\mathrm{Mg}, \mathrm{Si}$, and $\mathrm{Fe}$ ions.

Using values of $\mathrm{Fe}$ and $\mathrm{O}$ fluxes, we have calculated their abundance in SW for 8 energy ranges during spotless periods (Table 7).

The ACR effect results in minimum values of $\mathrm{Fe} / \mathrm{O}=0.004$ and 0.010 at $E_{\mathrm{k}}=5.3$ and $13.0 \mathrm{MeV} / \mathrm{n}$ respectively. Emphasize that when active regions appear on the Sun, energy spectra for all elements become similar and show a continuous rapid decrease in ion fluxes, especially at energies of hundreds of $\mathrm{MeV} / \mathrm{n}$. 
$\mathrm{Fe} / \mathrm{O}$ values in SW in 8 energy intervals in the absence of sunspots

\begin{tabular}{|c|c|c|c|c|c|c|c|c|}
\hline \multirow{2}{*}{$\begin{array}{c}\text { Periods of } \\
\text { absence of } \\
\text { sunspots }\end{array}$} & \multicolumn{8}{|c|}{$\mathrm{Fe} / \mathrm{O}$} \\
\hline & $\begin{array}{c}0.06 \\
\mathrm{MeV} / \mathrm{n}\end{array}$ & $\begin{array}{c}0.23 \\
\mathrm{MeV} / \mathrm{n}\end{array}$ & $\begin{array}{c}1.81 \\
\mathrm{MeV} / \mathrm{n}\end{array}$ & $\begin{array}{c}5.30 \\
\mathrm{MeV} / \mathrm{n}\end{array}$ & $\begin{array}{c}13.00 \\
\mathrm{MeV} / \mathrm{n}\end{array}$ & $\begin{array}{c}30.90 \\
\mathrm{MeV} / \mathrm{n}\end{array}$ & $\begin{array}{c}75.69 \\
\mathrm{MeV} / \mathrm{n}\end{array}$ & $\begin{array}{l}192.39 \\
\mathrm{MeV} / \mathrm{n}\end{array}$ \\
\hline $12.10-05.112007$ & 0.076 & 0.052 & 0.188 & 0.005 & 0.009 & 0.040 & 0.087 & 0.097 \\
\hline $27.09-10.102008$ & 0.079 & 0.055 & 0.109 & 0.007 & 0.016 & 0.072 & 0.093 & 0.107 \\
\hline 15.07-01.09 2009 & 0.075 & 0.045 & 0.152 & 0.001 & 0.005 & 0.048 & 0.086 & 0.099 \\
\hline Mean values & $\begin{array}{l}0.077 \pm \\
0.001\end{array}$ & $\begin{array}{l}0.051 \pm \\
0.003\end{array}$ & $\begin{array}{l}0.150 \pm \\
0.023\end{array}$ & $\begin{array}{l}0.004 \pm \\
0.002\end{array}$ & $\begin{array}{l}0.010 \pm \\
0.003\end{array}$ & $\begin{array}{l}0.053 \pm \\
0.010\end{array}$ & $\begin{array}{l}0.089 \pm \\
0.002\end{array}$ & $\begin{array}{l}0.101 \pm \\
0.003\end{array}$ \\
\hline
\end{tabular}

Table 8

Abundance of heavy elements vs oxygen for six periods of low solar activity

\begin{tabular}{|c|l|l|l|l|l|l|l|l|}
\hline $\begin{array}{c}\text { Periods of } \\
\text { low solar activity }\end{array}$ & \multicolumn{1}{|c|}{$\mathrm{Ne} / \mathrm{O}$} & $\mathrm{Mg} / \mathrm{O}$ & \multicolumn{1}{|c|}{$\mathrm{Si} / \mathrm{O}$} & \multicolumn{1}{|c|}{$\mathrm{S} / \mathrm{O}$} & \multicolumn{1}{|c|}{$\mathrm{Ar} / \mathrm{O}$} & \multicolumn{1}{c|}{$\mathrm{Ca} / \mathrm{O}$} & \multicolumn{1}{|c|}{$\mathrm{Fe} / \mathrm{O}$} & $\mathrm{Ni} / \mathrm{O}$ \\
\hline $22.07-25.07 .2007$ & 0.130 & 0.225 & 0.134 & 0.0227 & 0.0106 & 0.0194 & 0.101 & 0.00546 \\
\hline $13.10-17.10 .2007$ & 0.143 & 0.179 & 0.134 & 0.0212 & 0.00439 & 0.0192 & 0.0970 & 0.00454 \\
\hline $25.07-08.08 .2008$ & 0.163 & 0.214 & 0.147 & 0.0247 & 0.00683 & 0.0193 & 0.100 & 0.00455 \\
\hline $07.10-10.10 .2008$ & 0.156 & 0.185 & 0.149 & 0.0235 & 0.0113 & 0.0231 & 0.100 & 0.00463 \\
\hline $10.08-20.08 .2009$ & 0.156 & 0.196 & 0.146 & 0.0207 & 0.00963 & 0.0180 & 0.0930 & 0.00412 \\
\hline $05.09-13.09 .2009$ & 0.207 & 0.207 & 0.153 & 0.0279 & 0.00891 & 0.0174 & 0.104 & 0.00484 \\
\hline Mean values & $0.153 \pm$ & $0.201 \pm$ & $0.144 \pm$ & $0.0234 \pm$ & $0.00861 \pm$ & $0.0194 \pm$ & $0.0991 \pm$ & $0.00469 \pm$ \\
& 0.006 & 0.007 & 0.003 & 0.0011 & 0.00105 & 0.0008 & 0.0015 & 0.00018 \\
\hline
\end{tabular}

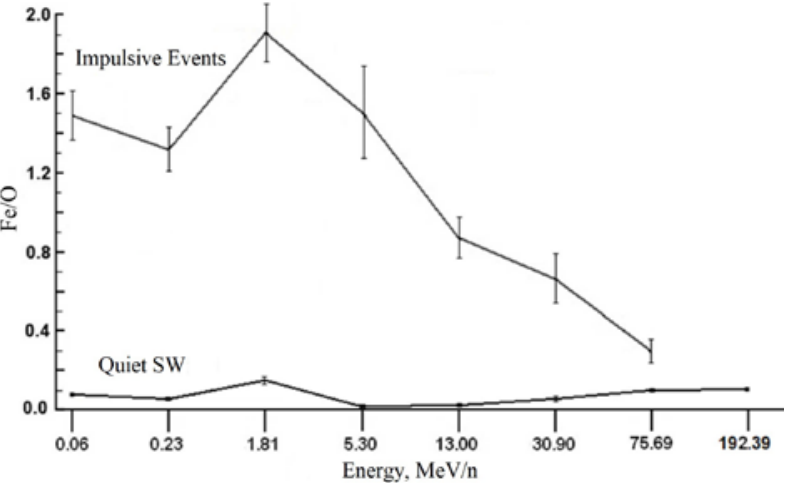

Figure 15. The Fe/O ratio by $1 \mathrm{AU}$ in the absence of sunspots vs particle fluxes from impulsive events for different energy intervals

Figure 15 presents the obtained curves of $\mathrm{Fe} / \mathrm{O}$ in SW and particle fluxes during the development of impulsive flare events in different ion energy ranges. The application of energy spectra of the $\mathrm{Fe}$ and $\mathrm{O}$ ions allowed us to use a fairly wide range of energies when calculating their abundances.

In impulsive events and SW in the absence of sunspots there are noticeable variations in $\mathrm{Fe} / \mathrm{O}$ with ion energies. There is a tendency for a substantial increase in $\mathrm{Fe} / \mathrm{O}$ in flare energetic fluxes on $\mathrm{Fe} / \mathrm{O}$ in $\mathrm{SW}$ at $E_{\mathrm{k}}<30 \mathrm{MeV} / \mathrm{n}$, becoming much smaller at higher ion energies. Fe/O curves for impulsive flare events and SW under conditions of minimum solar activity show similar variations in a lowenergy ion range $0.06-2.00 \mathrm{MeV} / \mathrm{n}$.

At $E_{\mathrm{k}}=1.81 \mathrm{MeV} / \mathrm{n}$, both the curves exhibit maximum $\mathrm{Fe} / \mathrm{O}$ values. Thus, despite the difference in absolute values of $\mathrm{Fe} / \mathrm{O}$, in impulsive flares during normal activity and under quiet conditions, processes occur on the Sun which lead to a relative increase in abundances of Fe with respect to $\mathrm{O}$ in SW.

\section{BEHAVIOR OF THE Fe/O RATIO IN GCR FLUXES IN THE ABSENCE OF DISTURBANCES IN THE SOLAR WIND DURING SOLAR MINIMUM}

It is of interest to study the behavior of GCR fluxes in the energy range $E_{\mathrm{k}}>30 \mathrm{MeV} / \mathrm{n}$ for different ions, including Fe and O. When studying GCR properties, we pay special attention to the identification of periods in which SW was under low solar activity with background parameter values. In addition to the requirements described in previous papers [Desai et al., 2006; Zeldovich et al., 2010] for selection of intervals, we adopt a more thorough approach. Thus we have chosen six SW observation periods when conditions of complete absence of sunspots were fulfilled and fast plasma flows from $\mathrm{CH}$ with the open structure of magnetic field lines had no effect. We also exclude the effect of various high-speed fluxes which are formed at the boundaries of sectors separating multipolar IMF regions, when SW moves through them. Thus we eliminate the influence of various high-speed fluxes $\left(V_{\mathrm{SW}}<480 \mathrm{~km} / \mathrm{s}\right)$ and particle fluxes with high density $\left(N_{\mathrm{p}}<16 \mathrm{~cm}^{-3}\right)$. Values of the averaged vector of IMF strength and its vertical component also do not exceed values corresponding to the field in SW plasma under similar conditions.

Such a detailed account of possible causes leading to activation of plasma flows allows us to study the composition of admixtures in SW plasma near the outer boundaries of Earth's magnetosphere (Table 8, column 1). It is known that GCRs consist largely of $\mathrm{H}(\sim 92 \%)$ and $\mathrm{He}$ ( $\sim$ \%) nuclei. At the same time, heavier nuclei (1\%) provide most of the information about the origin of CRs from data on their elemental and isotope composition. 


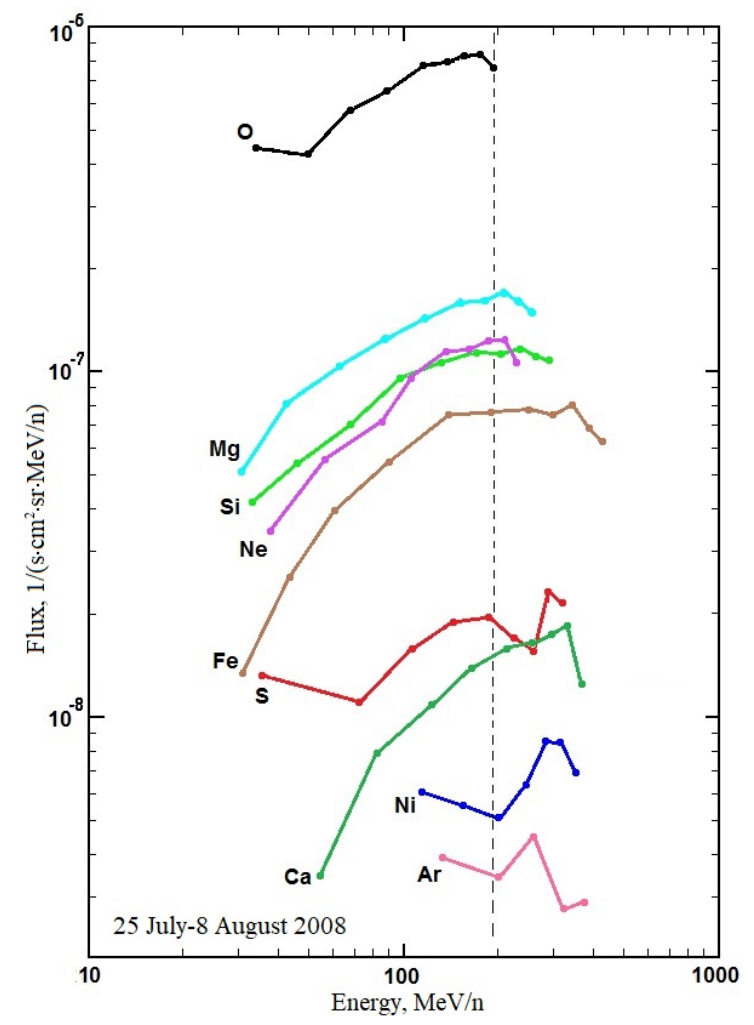

Figure 16. Energy spectra of nine elements in SW streams during low solar activity. An increase in ion fluxes in SW for $E_{\mathrm{k}}>30 \mathrm{MeV} / \mathrm{n}$ is largely determined by the presence of GCR. The vertical dashed line is $192.39 \mathrm{MeV} / \mathrm{n}$

Data from the ACE isotope spectrometer (CRIS) make it possible to study characteristics of the GCR nuclei in the energy range $E_{\mathrm{k}}=30 \div 500 \mathrm{MeV} / \mathrm{n}$. For the six selected intervals, we have constructed energy spectra of nine elements (O, Mg, Si, Ne, Fe, S, Ca, Ni, Ar) with the maximum presence of GCRs in SW. Figure 16 exemplifies GCR energy spectra for July 25 - August 8, 2008.

Energy spectra for all the elements are characterized by an increase in their values with peaks at $E_{\mathrm{k}} \sim 100 \div 300 \mathrm{MeV} / \mathrm{n}$. We also note the following regularity: the higher is the element charge, the higher are the energies at which a maximum GCR flux is observed. Most of the elements considered exhibit a relative enhancement of particle fluxes by about an order of magnitude in the GCR energy range.

Data on ion fluxes allow us to determine abundances of heavy elements. In this way, we determine it for $E_{\mathrm{k}}=192.39 \mathrm{MeV} / \mathrm{n}$ near which maximum fluxes with the largest abundance of heavy GCR elements occur. We calculate abundances of eight heavy elements with respect to oxygen.

Table 8 lists abundances of eight heavy GCR elements for $192.39 \mathrm{MeV} / \mathrm{n}$.

Abundances of each of the elements for all the six intervals at $E_{\mathrm{k}}=192.39 \mathrm{MeV} / \mathrm{n}$ are close in value, so the error in determining mean values is smaller than $\pm 5 \%$.

The results presented in Figure 16 allow us to conventionally divide the data on fluxes of heavy elements into two groups: $\mathrm{Ne}, \mathrm{Mg}, \mathrm{Si}$, and Fe having an abundance in the fluxes close to that of light elements ( $\mathrm{C}$ and $\mathrm{N}$ ), and S, Ar, Ca, Ni with much lower abundance.

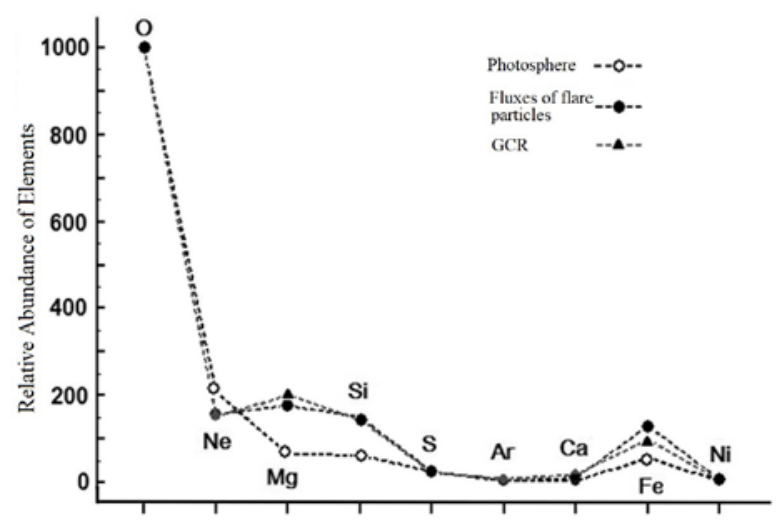

Figure 17. Comparison between abundances of elements in the photosphere in flare-induced fluxes and GCR

Figure 17 compare abundances of heavy elements in the GCR composition and in the solar photosphere and particle fluxes from gradual flares [Reames, 2014]. To facilitate the comparison with different structural formations, we refer all abundances of heavy elements to $\mathrm{O}$, the abundance of which is taken as 1000 .

The comparison between abundances of heavy elements in GCR and long-term flare-induced fluxes shows their good agreement. Thus, we have the following picture: during solar minimum and low geomagnetic activity, there are $30-500 \mathrm{MeV} / \mathrm{n}$ GCR fluxes with abundances of heavy elements similar to those in long-term flareinduced fluxes during high solar activity.

The Fe/O values calculated for fairly long periods without sunspots (Table 7) and for shorter intervals of low solar activity (Table 9) show good agreement in GCR energy intervals. Similar results are also obtained by using the $\mathrm{Fe}$ and $\mathrm{O}$ energy spectra constructed during integration over the entire solar minimum from April 1, 2007 to January 1, 2010. This suggests that a sufficient condition for the presence of GCR fluxes near Earth's orbit is the absence of active regions on the Sun as the main sources of disturbances in the medium, which prevent GCR propagation in the heliosphere.

As for the abundances of $\mathrm{Fe}$ and O, ACE (SIS, CRIS) observations show that at $E_{\mathrm{k}} \sim 200 \mathrm{MeV} / \mathrm{n}$ they practically coincide in particle fluxes from gradual flares and in GCR in the absence of sunspots. For more energetic particles, their abundance in GCR significantly exceeds that of flare particles of similar energies.

To explain the absence of noticeable disturbances in Earth's magnetosphere when GCRs are observed near its outer boundary, we should take into account the absence of directed fluxes of solar particles and the increased magnetic field strength in the presence of $B_{z}$. Under such conditions, interplanetary and magnetospheric magnetic fields do not reconnect; therefore there are no discontinuities in the outer boundary structure of the geomagnetosphere. This prevents GCR particles from penetrating inside. GCRs might lose energy during collisions with other particles, including those at the leading edge of the magnetosphere.

Thus, physical conditions in SW during solar minimum can be conventionally characterized by three basic states associated with 2-5 and 20-30 MeV/n particle energies. At $E_{\mathrm{k}} \leq 2 \mathrm{MeV} / \mathrm{n}, \mathrm{SW}$ conditions 
Fe/O index for particle fluxes with predominant influence of GCR in different energy intervals

\begin{tabular}{|c|c|c|c|c|c|}
\hline \multirow{2}{*}{$\begin{array}{c}\text { Periods of } \\
\text { low solar activity }\end{array}$} & \multicolumn{5}{|c|}{ Fe/O } \\
\cline { 2 - 6 } & $30.90 \mathrm{MeV} / \mathrm{n}$ & $49.82 \mathrm{MeV} / \mathrm{n}$ & $75.69 \mathrm{MeV} / \mathrm{n}$ & $140.39 \mathrm{MeV} / \mathrm{n}$ & $192.39 \mathrm{MeV} / \mathrm{n}$ \\
\hline $22.07-25.07 .2007$ & 0.101 & 0.082 & 0.087 & 0.094 & 0.101 \\
\hline $13.10-17.10 .2007$ & 0.033 & 0.122 & 0.063 & 0.083 & 0.097 \\
\hline $25.07-08.08 .2008$ & 0.032 & 0.074 & 0.092 & 0.097 & 0.100 \\
\hline $07.10-10.10 .2008$ & 0.064 & 0.072 & 0.090 & 0.092 & 0.100 \\
\hline $10.08-20.08 .2009$ & 0.042 & 0.081 & 0.095 & 0.095 & 0.093 \\
\hline $05.09-13.09 .2009$ & 0.054 & 0.088 & 0.100 & 0.086 & 0.104 \\
\hline Mean & $0.054 \pm 0.011$ & $0.086 \pm 0.007$ & $0.088 \pm 0.005$ & $0.091 \pm 0.002$ & $0.099 \pm 0.002$ \\
\hline
\end{tabular}

largely depend on solar activity; and at 2-30 MeV/n, on ACR. In this case, for SW plasma in the quiet state considered, an interesting effect may occur: abundance of ions of elements with higher FIP can exceed that of ions with lower FIP as compared to those under usual conditions (it should be noted that this conclusion is preliminary and requires confirmation).

Protons and other particles with $>30 \mathrm{MeV} / \mathrm{n}$ are generally of galactic origin [Garrard et al., 1997; Bazilevskaya et al., 2008]. Differences between Fe/O values in flare-induced fluxes of energetic particles and in SW at $E_{\mathrm{k}}<30 \mathrm{MeV} / \mathrm{n}$ reach $\sim 1.5$. For higher energies, under the dominant influence of GCR particle fluxes, this difference is much smaller. The small local maximum of the $\mathrm{Fe} / \mathrm{O}$ ratio (Figure 15) at ion energy of $\sim 1.81 \mathrm{MeV} / \mathrm{n}$ in the quiescent SW can be associated with the reconnection of magnetic fields at coronal bright points even during low solar activity. This process may cause the same manifestations of FIP effect as in impulsive solar flares.

Thus, the cause of the existence of the low minimum values $\mathrm{Fe} / \mathrm{O}=0.004$ and 0.010 at $E_{\mathrm{k}}=5.3$ and $13.0 \mathrm{MeV} / \mathrm{n}$ respectively is naturally explained. Emphasize that when active regions appear on the Sun, energy spectra for all the elements become similar and exhibit a continuous and rapid decrease in ion fluxes, especially in the energy range of the order of hundreds of $\mathrm{MeV} / \mathrm{n}$.

At solar minimum during low geomagnetic activity, there are 30-500 MeV/n GCR fluxes with abundances of heavy elements close to those in long-term flareinduced fluxes during high solar activity.

\section{STUDY OF COMPOSITION OF HIGH-SPEED SOLAR WIND STREAMS FROM CORONAL HOLES BASED ON Fe/O VARIATION IN VARIOUS ENERGY INTERVALS}

Interesting formations in the solar atmosphere in terms of fractionation of elements are CHs, whose main observable difference from surrounding regions of the quiet Sun is low emission in the UV and X-ray ranges of the electromagnetic spectrum in the corona.

During periods of decrease and minimum of solar activity, the role of CHs as sources of geomagnetic disturbances increases. It is considered an established fact that $\mathrm{CHs}$ are regions with open field lines along which plasma from the corona flows into SW. The darkening and contrast of CHs are attributed to the high-speed plasma outflows from them. This is confirmed by the findings reported in [Obridko et al., 2009].

The purpose of our study of CHs is to identify characteristics of admixtures in the composition of high-speed plasma flows producing magnetic storms of moderate power in Earth's magnetosphere. For this purpose, we have used satellite observations: X-ray filtergrams with $\mathrm{CH}$ images, time variations in various physical parameters of SW near Earth's orbit, as well as the geomagnetic index $D s t_{\text {min }}$ obtained from ground-based observations.

The comparison between Fe and $\mathrm{O}$ energy spectra in high-speed SW streams from equatorial $\mathrm{CHs}$ in the presence of active regions on the Sun and in their absence has shown significant differences not only in $\mathrm{Fe} / \mathrm{O}$ values and behavior, but also in the values of ion fluxes of different energies. The comparison of characteristics of the long-lived $\mathrm{CH}$ on June 22-23, 2013, which is characterized by a high-speed SW stream in the presence of sunspot groups ( $W=126)$, with the equatorial $\mathrm{CH}$ on September 22-25, 2007 in the absence of sunspots has revealed that the activity of sunspot groups causes the $\mathrm{Fe}$ and $\mathrm{O}$ fluxes to increase by almost two orders of magnitude in an energy range $0.06-1.0 \mathrm{MeV} / \mathrm{n}$. Thus, physical properties in plasma flows directly from the $\mathrm{CH}$ zone due to the influence of active regions are strongly veiled. Therefore, to study the $\mathrm{Fe} / \mathrm{O}$ behavior in the equatorial $\mathrm{CH}$, we have used periods without sunspots during solar minimum, but with magnetic storms of moderate power $\left(-50 \mathrm{nT}>D s t_{\min }>-100 \mathrm{nT}\right)$ in Earth's magnetosphere. It should be pointed out that the recorded high-speed flows from $\mathrm{CH}$ in the absence of sunspots have a physical structure belonging to the corona without the impact of flares and other active processes.

Figure 18 depicts time variations in various physical parameters in SW plasma flows on October 24-30, 2007 during the passage of high-speed fluxes from the equatorial $\mathrm{CH}$ and its related magnetic storm with $D s t_{\min }=-53 \mathrm{nT}$.

Five-minute variations in the following plasma parameters are presented: in the average value of the total magnetic field vector $B(\mathrm{nT})$; in the vertical component of the magnetic field $B_{\mathrm{z}}(\mathrm{nT})$; in temperature $T(\mathrm{~K})$, density $N_{\mathrm{p}}\left(\mathrm{cm}^{-3}\right)$, velocity $V(\mathrm{~km} / \mathrm{s})$, SW pressure $P_{\text {Sw }}(\mathrm{nPa})$, and hourly equatorial geomagnetic index Dst (nT). 


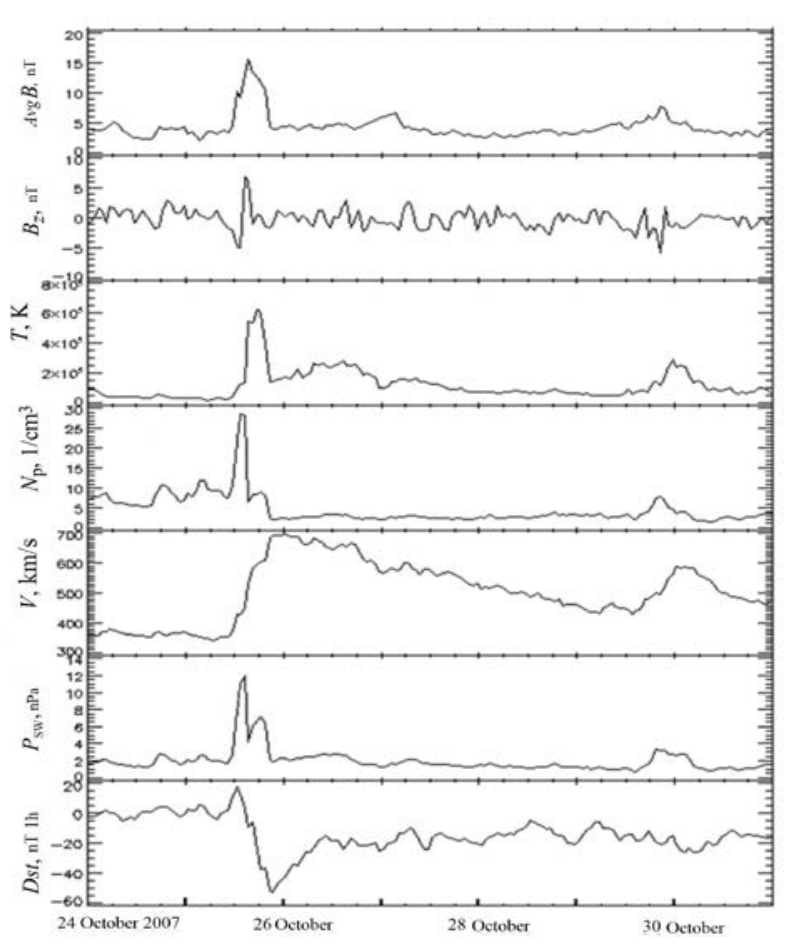

Figure 18. Change in physical parameters in the plasma flow from $\mathrm{CH}$, which caused a geomagnetic storm with $D s t_{\min }=-53 \mathrm{nT}$

The analysis of the time variations in the plasma parameters during the events considered shows that the arrival of high-speed plasma flows from $\mathrm{CH}$ at Earth's orbit is often accompanied by a shock front as is CME but with one significant difference. In plasma flows from $\mathrm{CH}$ there are jumps in the magnetic field strength, temperature, density, and gas pressure, but the velocity in the frontal region of the fluxes from $\mathrm{CH}$ increases gradually and reaches its maximum values only in $~ 1-3$ days; then it slowly decreases to background level. Thus, in Figure 18, the flux velocity reaches maximum when all other parameters have already exhibited jumps in the frontal region of the flux. In the plasma flow from $\mathrm{CH}$, only velocity is higher, whereas an increase in the remaining parameters is noticeable only in the frontal region of the flux.

A characteristic feature of the disturbed frontal region of the plasma flow is also the presence of the IMF southward component $B_{z}$ and density - another plasma parameter with high values. Both of these geoeffective parameters are often sources of geomagnetic storms.

Figure 19 compares $\mathrm{Fe}$ and $\mathrm{O}$ energy spectra during the passage of the entire flux from the October 24-30, $2007 \mathrm{CH}$ and separately of its frontal region representing the disturbed plasma region. For the total flux from $\mathrm{CH}$ at $E_{\mathrm{k}}=2 \div 30 \mathrm{MeV} / \mathrm{n}$, at which the ACR effect is most pronounced, the $\mathrm{Fe} / \mathrm{O}$ values decrease. Considering the flux from the disturbed frontal region separately, we can note a weak ACR effect that causes Fe/O to increase. This is mainly due to the relative increase in the Fe flux that has a lower FIP effect (Figure 19, b), and can occur because in the accelerated disturbed frontal region of the fluxes from CH there can arise Alfvén waves that lead to selective scattering of Fe and $\mathrm{O}$ ions subject to the FIP effect.

Table 10 lists calculated Fe/O values in eight energy intervals on the basis of the $\mathrm{Fe}$ and $\mathrm{O}$ energy spectra
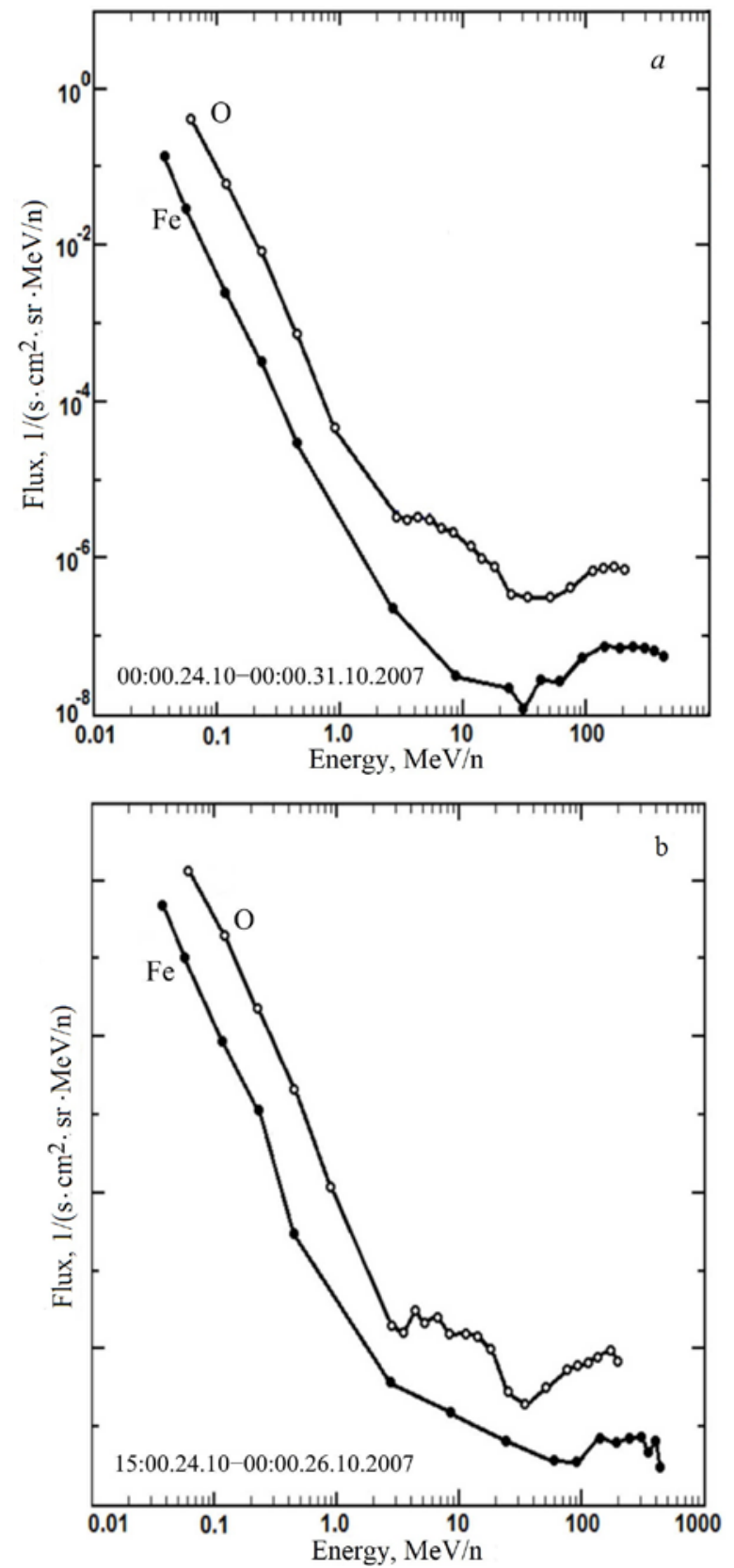

Figure 19. Fe and O energy spectra in the plasma flow from $\mathrm{CH}(a)$ and its disturbed frontal region $(b)$

constructed for seven accelerated geoeffective fluxes from CHs.

Thus, the most characteristic features of flows from $\mathrm{CHs}$, including the occurrence of geomagnetic storms, are associated with the disturbed frontal region of the fluxes. In the absence of sunspots, the behavior of $\mathrm{Fe} / \mathrm{O}$ for different ion energy ranges in the total flows from CHs and SW during solar minimum shows only minor deviations (Figure 20).

We can conventionally distinguish three groups of curves on the plots describing variations in the $\mathrm{Fe} / \mathrm{O}$ ratio with ion energy (Figure 20): 1 - fluxes from im-

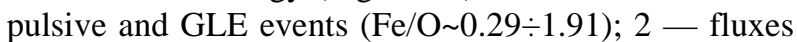
in $\mathrm{MC}$, from gradual flares, and in a shell between CME shock and $\mathrm{MC}(\mathrm{Fe} / \mathrm{O} \sim 0.11 \div 0.52) ; 3$ - in fluxes from $\mathrm{CH}$ during solar minimum and in the absence of sunspots $(\mathrm{Fe} / \mathrm{O}=0.004 \div 0.178)$. 
$\mathrm{Fe} / \mathrm{O}$ in plasma flows from $\mathrm{CH}$ as sources of geomagnetic storms in the absence of sunspots (ions from $\mathrm{CH}<3 \mathrm{MeV} / \mathrm{n}$, then ACR and GCR ions)

\begin{tabular}{|c|c|c|c|c|c|c|c|c|}
\hline \multirow{2}{*}{$\begin{array}{l}\text { Periods of particle } \\
\text { fluxes from CH } \\
\text { in the absence } \\
\text { of sunspots }\end{array}$} & \multicolumn{8}{|c|}{$\mathrm{Fe} / \mathrm{O}$} \\
\hline & $0.06 \mathrm{MeV} / \mathrm{n}$ & $0.23 \mathrm{MeV} / \mathrm{n}$ & $1.81 \mathrm{MeV} / \mathrm{n}$ & $.30 \mathrm{MeV} / \mathrm{n}$ & $13.00 \mathrm{MeV} / \mathrm{n}$ & $30.90 \mathrm{MeV} / \mathrm{n}$ & $\begin{array}{c}75.69 \\
\mathrm{MeV} / \mathrm{n}\end{array}$ & 192.39 MeV/r \\
\hline 21.03-27.03.2007 & 0.035 & 0.063 & 0.118 & 0.027 & 0.010 & 0.097 & 0.130 & 0.083 \\
\hline 31.03-04.04.2007 & 0.066 & 0.020 & 0.127 & 0.044 & 0.016 & 0.050 & 0.105 & 0.094 \\
\hline $24.10-30.10 .2007$ & 0.072 & 0.042 & 0.104 & 0.021 & 0.009 & 0.034 & 0.099 & 0.096 \\
\hline 19.11-27.11.2007 & 0.138 & 0.077 & 0.181 & 0.016 & 0.008 & 0.059 & 0.072 & 0.112 \\
\hline $07.03-13.03 .2008$ & 0.091 & 0.049 & 0.352 & 0.034 & 0.009 & 0.074 & 0.101 & 0.101 \\
\hline 02.09-09.09.2008 & 0.083 & 0.010 & 0.101 & 0.059 & 0.013 & 0.051 & 0.110 & 0.107 \\
\hline 19.07-26.07.2009 & 0.066 & 0.052 & 0.264 & 0.013 & 0.004 & 0.047 & 0.096 & 0.102 \\
\hline Mean values & $0.079 \pm 0.012$ & $0.054 \pm 0.012$ & $0.178 \pm 0.036$ & $0.031 \pm 0.006$ & $0.010 \pm 0.001$ & $0.059 \pm 0.008$ & $0.102 \pm 0.007$ & $0.099 \pm 0.004$ \\
\hline
\end{tabular}

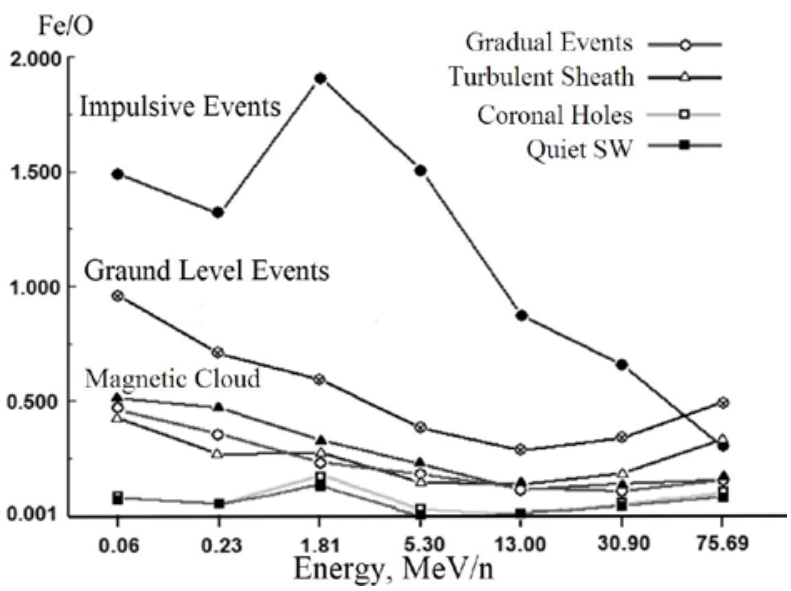

Figure 20. Comparison of the Fe/O behavior with ion energy for various active processes and during periods of solar minimum

The most noticeable increases in $\mathrm{Fe} / \mathrm{O}$ for $E_{\mathrm{k}}=1.81 \mathrm{MeV} / \mathrm{n}$ occur in particle fluxes from impulsive events, in a shell in front of MC, in plasma flows from $\mathrm{CHs}$, and, finally, in the absence of sunspots (Figure 20). The $\mathrm{Fe} / \mathrm{O}$ ratios, which do not show an increase in their values at $E_{\mathrm{k}}=1.81 \mathrm{MeV} / \mathrm{n}$, refer only to gradual events and geoeffective MCs.

\section{CONCLUSION}

The analysis of physical characteristics of plasma composition in different solar activity manifestations and in periods of their absence, and estimated abundances of $\mathrm{Fe}$ and $\mathrm{O}$ admixtures over a wide ion energy range allow us to draw the following conclusions.

It has been shown that spacecraft record maximum $\mathrm{Fe} / \mathrm{O}$ ratios in particle fluxes from impulsive flare events for ion energies $<2 \mathrm{MeV} / \mathrm{n}$ (the most significant manifestation of the FIP effect). In particle fluxes from gradual events, $\mathrm{Fe} / \mathrm{O}$ decreases smoothly with increasing ion energies and is noticeably inferior to $\mathrm{Fe} / \mathrm{O}$ in particle fluxes from impulsive flares.

Magnetic clouds as a separate type of CME are sources of strong geomagnetic storms and exhibit $\mathrm{Fe}$ abundances comparable to its abundance in fluxes from gradual events.

It has been established that the $\mathrm{Fe} / \mathrm{O}$ ratio allows us to detect penetration of energetic ions of flare plasma into the CME body during the initial phase of its development.

For enhanced high-energy proton fluxes ( $>60 \mathrm{MeV} / \mathrm{n}$ ), we have recorded sharp changes in ion energy spectra in the form of jumps of particle fluxes in an energy range $1.5-2.5 \mathrm{MeV} / \mathrm{n}$, which is typical only of very powerful $\mathrm{X}$ flare events followed by high-speed CME events. The $\mathrm{Fe} / \mathrm{O}$ ratio in the intensity jump in such events can exceed 1.

During solar minimum with complete absence of sunspots, the $\mathrm{Fe} / \mathrm{O}$ ratios during low solar activity show absolutely low values of $\mathrm{Fe} / \mathrm{O}=0.004 \div 0.010$ in an energy range from $2-5$ to $30 \mathrm{MeV} / \mathrm{n}$. This is due to the manifestation of the anomalous CR component during solar minimum.

Thus, the Fe/O ratio can serve as an important diagnostic parameter of plasma state both for low solar activity and for the development of various solar processes.

We are grateful to the teams involved in conducting ACE, SOHO, GOES, WIND experiments for providing experimental data published on the Internet, as well as experts of the OMNI Web Plus Browser website [https://omniweb.gsfc.nasa.gov/ftpbrow-ser/flux _spectr_m.html].

The work was carried out in accordance with the work schedule under Program No. BR05336383 MDAI RK.

\section{REFERENCES}

Altyntsev A.T., Banin V.G., Kuklin G.V., Tomozov V.M. Solnechnye vspyshki [Solar Flares]. Moscow: Nauka Publ., 1982. 246 p. (In Russian).

Barkhatov N.A., Revunova E.A., Vinogradov A.B. Evolution of orientation of solar wind magnetic clouds, and manifestation of seasonal dependence in their geomagnetic activity. Vestnik Nizhegorodskogo universiteta imeni N.I. Lobachevskogo [Vestnik of Lobachevsky University of Nizhni Novgorod]. 2014, no. 4 (1), pp. 106-113. (In Russian).

Barkhatov N.A., Vinogradov A.B., Levitin A.E., Revunova E.A. Geomagnetic substorm activity associated with magnetic clouds. Geomagnetism and Aeronomy. 2015, vol. 55, no. 5, pp. 596-602. DOI: 10.1134/S0016793215050023.

Bazilevskaya G.A., Stozhkov Yu.I. Energichnye chastitsy i kosmicheskie luchi: galakticheskie, geliosfernye i solnechnye kosmicheskie luchi. Plazmennaya geliofizika [Energetic particles and cosmic rays: galactic, heliospheric and solar cosmic raya]. Eds. Zeleny L.M., Veselovsky I.S. V. 1. Moscow, 2008. P. 345-357. (In Russian). 
Cliver E.W., Gopalswamy E., Webb D.F. History of research on solar energetic particle (SEP) events: The evolving paradigm. Proc. the 2008 IAU Symposium. No. 257. Universal Heliophysical Processes. 2009, pp. 401-412.

Desai M. I., Mason G. M., Mazur J.E., Dwyer J.R. Solar cycle variations in the composition of the suprathermal heavy-ion population near 1 AU. Astrophys. J. 2006, vol. 645, pp. L81-L84.

Dierckxsens M., Tziotziou K., Dalla S., Patsou I., Marsh M.S., Crosby N.B., Malandraki O., Tsiropoula G. Relationship between solar energetic particles and properties of flares and CME: statistical analysis of solar cycle 23 events. Solar Phys. 2015, vol. 290, no. 3. pp. 841-874. DOI: $10.1007 /$ s11207014-0641-4.

Fisk L.A., Kozlovsky B., Ramaty R. An interpretation of the observed oxygen and nitrogen enhancement in low energy cosmic rays. Astrophys. J. Let. 1974, vol. 190, pp. 35-38.

Garrard T.L., Christian E.R., Mewaldt R.A., Ormes J.F., Stone E.C. The advanced composition explorer mission. Proc. 25th International Cosmic Ray Conference. Durban, South Africa, 30 July - 6 August, 1997. 1997, vol. 1, pp. 105-108.

Gonzalez W.D., Tsurutani B.T., Clua de Gonzalez A.L. Interplanetary origin of geomagnetic storms. Space Sci. Rev. 1999, vol. 88, pp. 529-562. DOI: 10.1023/A:1005160129098.

Gosling J.T., Pizzo V.J. Formation and evolution of corotating interaction regions and their three-dimensional structure. Space Sci. Rev. 1999, vol. 89, pp. 21-52. DOI: 10.1023/A:1005291711900.

Kahler S.W., Cliver E.W., Tylka A.J., Dietrich W.F. A comparison of ground level event e/p and $\mathrm{Fe} / \mathrm{O}$ ratios with associated solar flare and CME characteristics. Space Sci. Rev. 2012 vol. 171 , no. $1-4$, pp. $121-139$. DOI: $10.1007 / s 11214-011-$ 9768-x.

Kallenrode M. B. Current views on impulsive and gradual solar energetic particle events. J. Phys. G: Nuclear and Particle Phys. 2003, vol. 29, pp. 965-981. DOI: 10.1088/09543899/29/5/316.

Kasinsky V.V., Tomozov V.M. Comparison of X-ray coronal structures with dynamics and morphology of the photospheric activity. Astronomicheskii tsirkulyar [Astronomical Circular]. 1974, no. 806, pp. 1-3. (In Russian).

Klecker B. Current understanding of SEP acceleration and propagation. J. Phys.: Conf. Ser. 2013, vol. 409, no. 1, pp. 1-15. DOI: 10.1088/1742-6596/409/1/012015.

Laming J.M. A unified picture of the first ionization potential and inverse first ionization potential effects. Astrophys. $J$. 2004, vol. 614, pp. 1063-1072. DOI: 10.1086/423780.

Li G., Zank G.P. Mixed particle acceleration at CMEdriven shocks and flares. Geophys. Res. Let. 2005, vol. 32, no. 2 , pp. L02101. DOI: 10.1029/2004GL021250.

Minasyants G.S., Minasyants T.M. Some peculiarities in dynamics of particle fluxes of solar cosmic rays. XVIII Vserossiskaya ezhegodnaya konferentsiya po fizike Solntsa "Solnechnaya I solnechno-zemnaya fizika - 2014" [Proc. XVIII National Conference on Solar Physics "Solar and SolarTerrestrial Physics - 2014]. Saint Petersburg, Pulkovo Observatory, 2014, pp. 287-290. (In Russian).

Minasyants G.S., Minasyants T.M., Tomozov V.M. On some features in dynamics of energy spectra in flare fluxes of solar cosmic rays. Izvestiya Krymskoi astrofizicheskoi observatorii [Bull. of the Crimean Astrophysical Observatory]. 2016a, vol. 112, no.1, pp. 71-77. (In Russian).

Minasyants G.S., Minasyants T.M., Tomozov V.M. Variations of $\mathrm{Fe} / \mathrm{O}$ ratio during the disturbed period of SCR flux development. Manifestation of FIP effect in SCR composition. Geomagnetizm i aeronomiya [Geomagnetism and Aeronomy] 2016b, vol. 56, no. 2, pp. 217-227. (In Russian).

Minasyants G.S., Minasyants T.M., Tomozov V.M. Dynamics of $\mathrm{Fe} / \mathrm{O}$ ratio during variations of ion energy in fluxes of accelerated solar particles. Geomagnetizm i aeronomiya [Geomagnetism and Aeronomy]. 2016c, vol. 56, no. 6, pp. 690-699. (In Russian).

Minasyants G.S., Minasyants T.M., Tomozov V.M. Fe/O ratio variations during the disturbed stage in the development of the solar cosmic ray fluxes. Manifestations of the first ionization potential effect in the solar cosmic ray composition. Geomagnetism and Aeronomy. 2016, vol. 56, no. 2, pp. 203-212. DOI: 10.1134/S0016793216020110.

Mordvinov A.V., Tomozov V.M., Fainshtein V.G. Effect of electron distribution function on dynamics of collisionless plasma expansion into background plasma of lower density. Prikladnaya mekhanika i tekhnicheskaya fizika [Applied Mechanics and Technical Physics]. 1985, no. 6, pp. 10-15. (In Russian).

Nitta N.V., Reames D.V., De Rosa M.L., Liu Y. Solar sources of impulsive solar energetic particle events and their magnetic field connection to the Earth. Astrophys. J. 2006, vol. 650, pp. 438-450. DOI: 10.1086/507442.

Obridko V.N., Shelting B.D., Livshits I.M., Askerov A.B. Correlation between contrast of coronal holes and solar wind characteristics. Astronomicheskii zhurnal [Astron. J.] 2009, vol. 86, no. 11, pp. 1125-1132. (In Russian).

Pallavicini R., Serio S., Vaiana G. A survey of soft X-ray limb flare images - The relation between their structure in the corona and other physical parameters. Astrophys. J. Part 1. 1977, vol. 216, pp. 108-122.

Priest E.R., Forbes T. Magnitnoe peresoedinenie. Magnitogidrodinamicheskaya teoriya i prilozheniya [Magnetic reconnection. Magnetohydrodynamic theory and applications]. Moscow, Fizmatlit Publ., 2005. 591 p. (In Russian).

Richardson I.G., Cane H.V. Near-Earth interplanetary coronal mass ejections during solar cycle 23 (1996-2009): Catalog and summary of properties. Solar Phys. 2010, vol. 264, pp. 189-237. DOI: 10.1007/s11207-010-9568-6.

Reames D.V., Meyer J.P., von Rosenvinge T.T. Energetic particle abundances in impulsive solar flare events. Astrophys. $J$. Suppl. Ser. 1994, vol. 90, pp. 649-667.

Reames D.V. Solar energetic particles: A paradigm shift. Rev. Geophys. 1995, vol. 33, no. S1, pp. 585-589. DOI: 10.1029/95RG00188.

Reames D.E. Energetic particles composition. Solar and galactic composition: A Joint SOHO/ACE Workshop. Bern, Switzerland, 6-9 March, 2001 (AIP Conf. Proc.) 2001, vol. 598, pp. 153-164.

Reames D.V. The two sources of solar energetic particles. Space Sci. Rev. 2013, vol. 175, no. 1, pp. 53-92. DOI: $10.1007 / \mathrm{s} 11214-013-9958-9$.

Reames D.V. Element abundances in solar energetic particles and the solar corona. Solar Phys. 2014, vol. 289, no. 3. pp. 977-993. DOI: 10.1007/s11207-013-0350-4.

Reames D.V., Ng C.K. Heavy-element abundances in solar energetic particle events. Astrophys. J. 2004, vol. 610, no. 1. pp. 510-522. DOI: 10.1086/421518.

Roth I., Temerin M. Selective ion acceleration in impulsive solar flares. Adv. Space Res. 1998, vol. 21, pp. 591-595.

Somov B.V. Plasma Astrophysics: Reconnection and Flares. Springer: New York. 2013. 504 p. DOI: 10.1007/978-0-38768894-7.

Tomozov V.M. FIP effect as an indicator of dynamic processes in the solar atmosphere and interplanetary medium. Solnechno-zemnaya fizika [Solar-Terrestrial Physics]. 2012, iss. 19, pp. 19-35. (In Russian).

Tomozov V.M. On some regularities in chemical composition distribution in stellar atmospheres. Solnechnozemnaya fizika [Solar-Terrestrial Physics]. 2013, iss. 23, pp. 23-32. (In Russian).

Tomozov V.M., Strokin N.A. Comparative analysis of effectiveness of proton and electron acceleration in laboratory 
and solar plasma. Geomagnetizm i aeronomiya [Geomagnetism and Aeronomy]. 2015, vol. 55, no. 2, pp. 161-167. (In Russian) DOI: 10.7868/ S0016794015020169.

Torsti J., Kocharov L., Innes D.E., Laivola J., Sahla T. Injection of energetic protons during solar eruption on 1999 May 9: Effect of flare and coronal mass ejection. Astron. Astrophys. 2001, vol. 365, pp. 198-203. DOI: $10.1051 / 0004-$ 6361:20000148

Tylka A.J., Cohen C.M.S., Dietrich W.F., Lee M.A., Maclennan C.G., Mewaldt R.A., Ng C.K., Reames D.V. Shock geometry, seed populations and the origin of variable elemental composition at high energies in large gradual solar particle events. Astrophys. J. 2005, vol. 625, no. 1, pp. 474-495. DOI: $10.1086 / 429384$.

van Driel-Gesztelyi L., Culhane J.L. Magnetic flux emergence, activity, eruptions and magnetic clouds: following magnetic field from the Sun to the heliosphere. Space Sci. Rev. 2009, vol. 144, iss. 1, pp. 351-381. DOI: 10.1007/s11214008-9461-x.

Wang Y.-M, Pick M., Mason G.M. Coronal holes, jets and the origin of ${ }^{3} \mathrm{He}-\mathrm{rich}$ particle events. Astrophys. J. 2006, vol. 639, no. 1, pp. 495-509. DOI: 10.1086/499355.

Yermolaev Yu.I., Yermolaev M.Yu. Solar and interplanetary sources of geomagnetic storms: space weather aspects. Geophys. Processes and Biosphere. 2009, vol. 8, no. 1, pp. 5-35. (In Russian).

Yutian Chi, Chenglong Shen, Yuming Wang, Pinzhong Ye, Wang S. Statistical Study of the Interplanetary Coronal Mass Ejections from 1996 to 2014. arXiv: 1504.07849v1 [astroph.SR] 29 Apr 2015 P.1-9.
Zeldovich M.A., Ishkov V.N., Logachev Yu.I., Kechkemeti $\mathrm{K}$. Ion composition of low-energy particle fluxes at 1 a.u. during quiet period of solar activity. 31 Vseros iskaya konferentsiya po kosmicheskim lucham [31st Natic al Conference on Cosmic Rays]. Moscow, MSU, 2010, pp. 1-7. (In Russian).

Zhang J., Dere K.P., Howard R.A., Kundu M.R., White S.M. On the temporal relationship between coronal mass ejections and flares. Astrophys. J. 2001, vol. 559, no. 1, pp. 452-462. DOI: $10.1086 / 322405$.

Zurbuchen T.H., Weberg M., von Steiger R., Mewaldt R.A., Lepri S.T., Antiochos S.K. Composition of coronal mass ejections. Astrophys. J. 2016, vol. 826, no. 10, 8 p. DOI: $10.3847 / 0004-637 \mathrm{X} / 826 / 1 / 10$.

URL: https://omniweb.gsfc.nasa.gov/ftpbrowser/flux_spectr_ m.html (accessed 18.10.2017).

URL: http://www.srl.caltech.edu/sampex/Data-Center/DATA/EventSpectra (accessed 18.10.2017).

URL: https://wind.nasa.gov/fullcatalogue.php (accessed 18.10.2017).

How to cite this article

Minasyants G.S., Minasyants T.M., Tomozov V.M. Fe/O ratio behavior as an indicator of solar plasma state at different solar activity manifestations and in periods of their absence. Solar-Terrestrial Physics. 2018, vol. 4, no. 1, pp. 29-50. DOI: $10.12737 /$ stp-41201804. 